TRANSACTIONS OF THE

AMERICAN MATHEMATICAL SOCIETY

Volume 352, Number 6, Pages 2737-2764

S 0002-9947(99)02457-5

Article electronically published on October 21, 1999

\title{
QUADRATIC INTEGRAL GAMES AND CAUSAL SYNTHESIS
}

\author{
YUNCHENG YOU
}

\begin{abstract}
The game problem for an input-output system governed by a Volterra integral equation with respect to a quadratic performance functional is an untouched open problem. In this paper, it is studied by a new approach called projection causality. The main result is the causal synthesis which provides a causal feedback implementation of the optimal strategies in the saddle point sense. The linear feedback operator is determined by the solution of a Fredholm integral operator equation, which is independent of data functions and control functions. Two application examples are included. The first one is quadratic differential games of a linear system with arbitrary finite delays in the state variable and control variables. The second is the standard linearquadratic differential games, for which it is proved that the causal synthesis can be reduced to a known result where the feedback operator is determined by the solution of a differential Riccati operator equation.
\end{abstract}

\section{INTRODUCTION}

The state space approach (or called the time domain approach) provides an elegant mathematical framework for modern systems theory, control theory, and differential game theory. In this framework, many dynamical problems governed by ordinary differential equations, functional differential equations, partial differential equations, and stochastic differential equations can be formulated and studied in a unified manner via the operator semigroup theory and the mild solution formula. This approach has been richly developed to deal with many control problems and differential game problems of distributed parameter systems. The early results of infinite dimensional differential games, especially the well-posed linear quadratic differential games, can be found in [2], [3, [6], [9], and [10].

On the other hand, the frequency domain approach was rooted in the engineering control theory and features a direct processing of input-output relations in terms of transfer functions, no matter whether or not there exists a differential equation of state variables. State variables could be purely mathematical variables rather than physical quantities. As a matter of fact, this simplicity in modelling is a pragmatic merit of the frequency domain approach, which has been inherited by the $H^{\infty}$ optimization and robust control theory, cf. 1] and the references therein.

A universal linear input-output relation is described by a Volterra integral equation, which may or may not be the solution of an initial value problem for a linear evolutionary equation in a Banach space. For such a general linear dynamical

Received by the editors April 29, 1996 and, in revised form, April 1, 1998.

1991 Mathematics Subject Classification. Primary 90D25, 49N35; Secondary 45D05, 47N70, 49N55, 93B36.

Key words and phrases. Volterra integral equation, quadratic game, optimal strategy, projection causality, output feedback, Fredholm operator equation. 
model without the semigroup property, many issues concerning optimal controls, controllability, stabilization, and game problems remain open and challenging. The essential difficulty in dealing with such an integral model is that one simply cannot apply most of the effective techniques in dealing with various differential equations.

In this paper, we shall investigate the quadratic game problem of a linear Volterra integral system in Hilbert spaces. The principal objective is to find a causal synthesis of the optimal strategy pair of this problem by a new methodology called projection causality. Indeed, this method is a substantial generalization of the semicausality approach initiated in [4] and [5].

We shall denote by $L\left(X_{1}, X_{2}\right)$ the space of bounded linear operators from Banach space $X_{1}$ to Banach space $X_{2}$, with the operator norm. If $X_{1}=X_{2}$, then this operator space will be denoted by $L\left(X_{1}\right)$.

Let $T>0$ be finite and fixed. Let $Y, U$, and $V$ be real Hilbert spaces. Let $\Omega=\{(t, s) \in[0, T] \times[0, T]: 0 \leq s \leq t \leq T\}$. To introduce the quadratic integral game problem, consider an input-output relation given by the following Volterra integral equation,

$$
y(t)=f(t)+\int_{0}^{t}[G(t, s) u(s)+K(t, s) v(s)] d s, \quad t \in[0, T] .
$$

We shall make assumptions [A1] through [A4] below and later on.

[A1] Assume that $f \in L^{\infty}([0, T] ; Y)$.

[A2] Assume that $G \in C(\Omega ; L(U, Y))$ and $K \in C(\Omega ; L(V, Y))$, where the continuity is in the strong sense that for each $\xi \in U$ and $\zeta \in V$, the functions $G(t, s) \xi: \Omega \rightarrow Y$ and $K(t, s) \zeta: \Omega \rightarrow Y$ are strongly continuous. Moreover, as a convention, we assume that $G(t, s) \equiv 0$ and $K(t, s) \equiv 0$ whenever $(t, s) \in \mathbb{R}^{2} \backslash \Omega$.

Denote by $\boldsymbol{Y}=L^{2}(0, T ; Y), \boldsymbol{U}=L^{2}(0, T ; U)$, and $\boldsymbol{V}=L^{2}(0, T ; V)$. These function spaces can be called fibre spaces corresponding to their respective underlying spaces $Y, U$, and $V$. Set a quadratic performance functional

$$
J(u, v)=\langle M y(T), y(T)\rangle+\int_{0}^{t}\left[\langle Q y(t), y(t)\rangle+\left\langle R_{1} u(t), u(t)\right\rangle+\left\langle R_{2} v(t), v(t)\right\rangle\right] d t,
$$

where the inner-products $\langle\cdot, \cdot\rangle$ are with respect to $Y, U$, or $V$ according to the context. This integral game involves two players whose control variables are $u$ and $v$ respectively, $u$ being a minimizer of $J(u, v)$ while $v$ being a maximizer of $J(u, v)$. Here is one more assumption.

[A3] Assume that $M$ and $Q \in L(Y), R_{1} \in L(U)$, and $R_{2} \in L(V)$ are all selfadjoint, in addition, $R_{1}$ is coercively positive and $R_{2}$ is coercively negative. Hence, there are constants $\delta_{1}>0$ and $\delta_{2}>0$ such that $R_{1} \geq \delta_{1} I_{U}$ and $R_{2} \leq-\delta_{2} I_{V}$, with $I_{U}$ and $I_{V}$ being the identity operators on $U$ and $V$ respectively.

The integral game problem is to find an optimal strategy pair $(\hat{u}, \hat{v}) \in \boldsymbol{U} \times \boldsymbol{V}$ in the saddle point sense:

$$
J(\hat{u}, v) \leq J(\hat{u}, \hat{v}) \leq J(u, \hat{v}), \quad \text { for any } u \in \boldsymbol{U} \text { and any } v \in \boldsymbol{V} .
$$

The ultimate goal of this paper is to find a pair of feedback optimal strategies for the two players in the causal sense: namely, the determining expression of the optimal strategy pair $(u(t), v(t))$ at real time $t \in[0, T]$ in terms of the corresponding output $y$ should not involve any anti-causal future information of $y(s), s>t$. 
As an important remark at the first place, it will be naive and impossible to seek for such an implementation of the optimal strategy pair that the real time control values $u(t)$ and $v(t)$ only depend on the information of the data function $f(s), 0 \leq s \leq t$. This is because the real time values of the optimal strategy pair are determined not by any segment information of the data function $f$, but by the entire data function $f(t), t \in[0, T]$.

The results of this work are related to the $H^{\infty}$ optimization and robust control problems. As shown in [1] and the references therein, $H^{\infty}$ optimization problems in multivariable systems (or even infinite dimensional systems) can be solved in terms of a pair of operator Riccati equations. These Riccati equations have a flavor of the solution to a linear-quadratic differential game, with the designer as one player seeking for minimizing the cost functional, while the disturbance or other interference such as the uncertainty as the other fictitious player to maximize the cost. 1 also presented the differential game approach to the full information robust control problems. Indeed, the differential game approach (especially with quadratic performances) serves as the time domain interpretation of $H^{\infty}$ optimization problems and has some computational and conceptional benefits, provided that the underlying linear system can be expressed as an evolutionary differential equations. In general, dynamical and causal linear systems may not be modeled as differential equations. But they can always be modeled as Volterra integral equations by theoretical or experimental analysis. In that case, the quadratic integral games considered in this paper will serve as a time-domain approach to the corresponding $H^{\infty}$ problems, and the synthesis equations obtained in terms of Fredholm integral equations in this paper provide a new foundation for working on $H^{\infty}$ control problems of more general linear systems.

This paper is a substantial extension of the co-authored (A. J. Pritchard and Y. You) work in $[8]$ on the causal feedback optimal control for Volterra integral systems. The relationship between [8] and this paper is an analogue of the LQ optimal control versus the LQ differential game. The quadratic integral game of this paper has two new features. First, the cost functional has no convexity nor concavity, which causes the difficulty in the inversion of the parametrized performance operator and the inversion of the $\xi$-evolutionary operator in achieving the causal feedback optimal strategies. Second, the two-player nature of the game problem demands more delicate manipulations of all the involved operator matrices. On the other hand, the common feature of the results in $[8]$ and in this paper is the same type of synthesis equations: the Fredholm integral operator equations determined only by the structural data of the Volterra integral system and independent of two players' strategies.

Before tackling this problem, let us make an observation that more general Volterra integral equations in the form of

$$
y(t)=f(t)+\int_{0}^{t}[\Lambda(t, s) y(s)+G(t, s) u(s)+K(t, s) v(s)] d s, \quad t \in[0, T],
$$

where $\Lambda \in C(\Omega ; L(Y))$, can be reduced to the form (1.1) with the above three assumptions satisfied. This reduction is via the resolvent operator as described by the following lemma, whose proof is a routine procedure of inversion in functional analysis. 
Lemma 1.1. The linear operator $\vartheta \in L(C([0, T] ; \boldsymbol{Y}))$ defined by

$$
(\vartheta y)(t)=\int_{0}^{t} \Lambda(t, s) y(s) d s, \quad t \in[0, T]
$$

is a quasi-nilpotent operator with the spectrum $\sigma(\vartheta)=\{0\}$. There exists a unique solution $y$ of the integral equation (1.4), which is expressed by

$$
y(t)=h(t)+\int_{0}^{t}[\widetilde{G}(t, s) u(s)+\widetilde{K}(t, s) v(s)] d s, \quad t \in[0, T],
$$

where

$$
\widetilde{G}(t, s)=G(t, s)+\int_{0}^{t} R(t, s) G(t, s) d s \text { and } \widetilde{K}(t, s)=K(t, s)+\int_{0}^{t} R(t, s) K(t, s) d s,
$$

in which the resolvent kernel $R(t, s)$ is given by

$$
R(t, s)=\sum_{j=1}^{\infty} \Lambda_{j}(t, s)
$$

which converges in the operator norm, with

$$
\Lambda_{1}(t, s)=\Lambda(t, s), \quad \Lambda_{j+1}(t, s)=\int_{0}^{t} \Lambda(t, \rho) \Lambda_{j}(\rho, s) d \rho, \quad j=1,2, \ldots .
$$

Note that by the Banach-Steinhaus theorem, [A2] implies that

$$
G_{0}=\sup \{\|G(t, s)\|:(t, s) \in \Omega\}<\infty \text { and } K_{0}=\sup \{\|K(t, s)\|:(t, s) \in \Omega\}<\infty .
$$

The rest of the paper is outlined as follows. We start with the existence and the open-loop result of the optimal strategy in Section 2 then we shall introduce the pivot concepts of projection causality with the tool lemmas in Section 3 After that, we shall prove the abstract synthesis principle at the level of the fibre spaces in Section 4. Then in Section 5, we introduce the parametrized Fredholm integral equation whose solution will yield the feedback operator in the causal synthesis. The proof of the existence and uniqueness of a strongly continuous solution to this Fredholm integral equation is given. In Section 6, the Fredholm inversion gives rise to the final result on the real-time synthesis of the optimal strategy pair at the level of the underlying spaces. In Section 7 we shall illustrate the application of the obtained result to the quadratic differential game of linear delay systems. Finally in Section 8, we shall show that, for the standard linear quadratic differential game, the new result can be reduced to the well-known synthesis whose feedback operator is given by the solution of the associated differential Riccati equation.

\section{The EXistence of optimal StRAtegy}

In this section, we shall prove the existence of optimal strategy under a wellposed assumption [A4]. The derived optimality condition provides an open-loop expression of an optimal strategy pair. 
Define following bounded linear operators on the fibre spaces $\boldsymbol{Y}, \boldsymbol{U}$, and $\boldsymbol{V}$ :

$$
\begin{aligned}
(\Gamma u)(t) & =\int_{0}^{t} G(t, s) u(s) d s, \quad t \in[0, T], \text { for } u \in \boldsymbol{U} ; \\
\Gamma_{T} u & =\int_{0}^{T} G(T, s) u(s) d s=(\Gamma u)(T), \text { for } u \in \boldsymbol{U} ; \\
(\Delta u)(t) & =\int_{0}^{t} K(t, s) v(s) d s, \quad t \in[0, T], \text { for } v \in \boldsymbol{V} ; \\
\Delta_{T} u & =\int_{0}^{T} K(T, s) v(s) d s=(\Delta u)(T), \text { for } v \in \boldsymbol{V} ; \\
(\boldsymbol{Q} y)(t) & =Q y(t), \quad t \in[0, T], \text { for } y \in \boldsymbol{Y} ; \\
\left(\mathbb{R}_{1} u\right)(t) & =R_{1} u(t), \quad t \in[0, T], \text { for } u \in \boldsymbol{U} ; \\
\left(\mathbb{R}_{2} v\right)(t) & =R_{2} v(t), \quad t \in[0, T], \text { for } v \in \boldsymbol{V} .
\end{aligned}
$$

It is easy to see that $\Gamma \in L(\boldsymbol{U} ; \boldsymbol{Y}), \Gamma_{T} \in L(\boldsymbol{U} ; Y), \Delta \in L(\boldsymbol{V} ; \boldsymbol{Y}), \Delta_{T} \in L(\boldsymbol{V} ; Y)$, $\boldsymbol{Q} \in L(\boldsymbol{Y}), \mathbb{R}_{1} \in L(\boldsymbol{U})$, and $\mathbb{R}_{2} \in L(\boldsymbol{V})$. Moreover, by assumption [A3], $\boldsymbol{Q}$ is self-adjoint, $\mathbb{R}_{1}$ is coercively positive and $\mathbb{R}_{2}$ is coercively negative. The adjoint operators of $\Gamma, \Gamma_{T}, \Delta$, and $\Delta_{T}$ are given by, respectively,

$$
\begin{aligned}
\left(\Gamma^{*} y\right)(t) & =\int_{t}^{T} G^{*}(\tau, t) y(\tau) d \tau, \quad t \in[0, T], \text { for } y \in \boldsymbol{Y} \\
\left(\Gamma_{T}^{*} \xi\right)(t) & =G^{*}(T, t) \xi, \quad t \in[0, T], \text { for } \xi \in Y ; \\
\left(\Delta^{*} y\right)(t) & =\int_{t}^{T} K^{*}(\tau, t) y(\tau) d \tau, \quad t \in[0, T], \text { for } y \in \boldsymbol{Y} ; \\
\left(\Delta_{T}^{*} \xi\right)(t) & =K^{*}(T, t) \xi, \quad t \in[0, T], \text { for } \xi \in Y .
\end{aligned}
$$

Following operators $\Phi \in L(\boldsymbol{U}), S \in L(\boldsymbol{U}, \boldsymbol{V}), \Psi \in L(\boldsymbol{V})$, and $W \in L(\boldsymbol{U} \times \boldsymbol{V})$ will play important roles in the mathematical treatment of this game problem:

$$
\begin{aligned}
\Phi & =\mathbb{R}_{1}+\Gamma^{*} \boldsymbol{Q} \Gamma+\Gamma_{T}^{*} M \Gamma_{T}, \\
S & =\Gamma^{*} \boldsymbol{Q} \Delta+\Gamma_{T}^{*} M \Delta_{T}, \\
\Psi & =\mathbb{R}_{2}+\Delta^{*} \boldsymbol{Q} \Delta+\Delta_{T}^{*} M \Delta_{T}, \\
W & =\left[\begin{array}{cr}
\Phi & S \\
S^{*} & \Psi
\end{array}\right] .
\end{aligned}
$$

Of course, $\Phi, \Psi$, and $W$ are self-adjoint operators. Below is the last assumption of this integral game problem:

[A4] Assume that $\Phi>0$ (coercively positive) and $\Psi<0$ (coercively negative). Thus, there is a positive constant $\varepsilon>0$ such that $\Phi \geq \varepsilon I$ and $\Psi \leq-\varepsilon I$, with $I$ the identity operator on $\boldsymbol{U}$ and on $\boldsymbol{V}$, respectively.

We have a justifying remark on this assumption [A4]. Similar to Theorem 2 in [9, it can be shown that a necessary condition for the existence of an optimal strategy pair in the saddle point sense is that

$$
\Phi \geq 0 \text { and } \Psi \leq 0 .
$$

Therefore, the assumption [A4] as a slight strengthening of the necessary condition (2.4) is reasonable. The following lemma on the invertibility of the operator $W$ will be used in the proof of the existence of optimal strategy. 
Lemma 2.1. Under the assumption [A4], the operator $W$ admits a bounded inverse $W^{-1} \in L(\boldsymbol{U} \times \boldsymbol{V})$, which is expressed by

$$
W^{-1}=\left[\begin{array}{cc}
A^{-1} & -A^{-1} S \Psi^{-1} \\
-B^{-1} S^{*} \Phi^{-1} & B^{-1}
\end{array}\right],
$$

where

$$
A=\Phi-S \Psi^{-1} S^{*} \quad \text { and } \quad B=\Psi-S^{*} \Phi^{-1} S .
$$

Proof. Since $A \geq \Phi>0$ and $B \leq \Psi<0$, the operators $A$ and $B$ are both boundedly invertible, the operator at the right-hand side of (2.5) (denoted by $W^{\#}$ ) is welldefined and belongs to $L(\boldsymbol{U} \times \boldsymbol{V})$. It is easy to check that this operator $W^{\#}$ is the left inverse operator of $W$ :

$$
\begin{gathered}
A^{-1} \Phi-A^{-1} S \Psi^{-1} S^{*}=A^{-1} \Phi-A^{-1}\left(S \Psi^{-1} S^{*}-\Phi+\Phi\right)=I_{U}, \\
A^{-1} S-A^{-1} S \Psi^{-1} \Psi=0, \\
-B^{-1} S^{*} \Phi^{-1} S+B^{-1} \Psi=-B^{-1}\left(S^{*} \Phi^{-1} S-\Psi+\Psi\right)+B^{-1} \Psi=I_{V} .
\end{gathered}
$$

Since $W$ is a self-adjoint and bounded operator, and $W^{\#} W=I$, we have

$$
I=\left(W^{\#} W\right)^{*}=W^{*}\left(W^{\#}\right)^{*}=W\left(W^{\#}\right)^{*},
$$

so that the right inverse of $W$ also exists. Therefore, it holds that $W^{\#}=\left(W^{\#}\right)^{*}=$ $W^{-1}$, which is the inverse operator of $W$.

Let us make a convention that the standing assumptions mean [A1] through [A4]. In the rest of this paper, as long as there is no confusion, we shall drop the notation ${ }^{\wedge}$ of the optimal strategy pair. The output function $y(\cdot)$ corresponding to a strategy pair $(u, v)$ will be denoted by $y(u, v)$.

Theorem 2.2 (Existence and Characterization). Under the standing assumptions, there exists a unique optimal strategy pair $(u, v)$ for this integral game problem. A game process $\{u, v, y(u, v)\}$ is optimal if and only if the following relations are satisfied,

$$
\begin{aligned}
& u(t)=-R_{1}^{-1}\left[G^{*}(T, t) M y(T)+\int_{t}^{T} G^{*}(s, t) Q y(s) d s\right], \quad t \in[0, T] \\
& v(t)=-R_{2}^{-1}\left[K^{*}(T, t) M y(T)+\int_{t}^{T} K^{*}(s, t) Q y(s) d s\right], \quad t \in[0, T] .
\end{aligned}
$$

Proof. In the performance functional (1.2), we can make substitutions

$$
y=f+\Gamma u+\Delta v \text { and } y(T)=f(T)+\Gamma_{T} u+\Delta_{T} v,
$$

to obtain a quadratic form on the Hilbert space $\boldsymbol{U} \times \boldsymbol{V}$,

$$
\begin{aligned}
J(u, v)= & \left\langle M\left(f(T)+\Gamma_{T} u+\Delta_{T} v\right), f(T)+\Gamma_{T} u+\Delta_{T} v\right\rangle \\
& +\langle\boldsymbol{Q}(f+\Gamma u+\Delta v), f+\Gamma u+\Delta v\rangle+\left\langle\mathbb{R}_{1} u, u\right\rangle+\left\langle\mathbb{R}_{2} u, u\right\rangle \\
= & \langle\Phi u, u\rangle+\langle\Psi v, v\rangle+2\left[\langle\boldsymbol{Q} \Gamma u, \Delta v\rangle+\left\langle M \Gamma_{T} u, \Delta_{T} v\right\rangle\right] \\
& +2\left\langle u, \Gamma^{*} \boldsymbol{Q} f+\Gamma_{T}^{*} M f(T)\right\rangle \\
& +2\left\langle v, \Delta^{*} \boldsymbol{Q} f+\Delta_{T}^{*} M f(T)\right\rangle+[\langle M f(T), f(T)\rangle+\langle\boldsymbol{Q} f, f\rangle]
\end{aligned}
$$


From (2.12) and [A4], we see that for a relatively fixed $v \in \boldsymbol{V}$, the minimizer $u$ for $J(u, v)$ exists uniquely and is given by

$$
\Phi u+\Gamma^{*} \boldsymbol{Q}[\Delta v+f]+\Gamma_{T}^{*} M\left[\Delta_{T} v+f(T)\right]=0,
$$

so that by (2.3) and (2.11), we have

$$
\begin{aligned}
\mathbb{R}_{1} u & =-\left\{\Gamma^{*} \boldsymbol{Q}[\Gamma u+\Delta v+f]+\Gamma_{T}^{*} M\left[\Gamma_{T} u+\Delta_{T} v+f(T)\right]\right\} \\
& =-\left\{\Gamma^{*} \boldsymbol{Q} y+\Gamma_{T}^{*} M y(T)\right\} .
\end{aligned}
$$

Similarly, for a relatively fixed $u \in \boldsymbol{U}$, the maximizer $v$ for $J(u, v)$ exists uniquely and is given by

$$
\begin{aligned}
\mathbb{R}_{2} v & =-\left\{\Delta^{*} \boldsymbol{Q}[\Gamma u+\Delta v+f]+\Delta_{T}^{*} M\left[\Gamma_{T} u+\Delta_{T} v+f(T)\right]\right\} \\
& =-\left\{\Delta^{*} \boldsymbol{Q} y+\Delta_{T}^{*} M y(T)\right\} .
\end{aligned}
$$

Moreover, we have to prove that the strategy pair $(u, v)$ implicitly defined by (2.14) and (2.15) does exist in $\boldsymbol{U} \times \boldsymbol{V}$. In fact, the coupled equations (2.14) and (2.15) can be rewritten as

$$
W\left(\begin{array}{l}
u \\
v
\end{array}\right)=-\left[\begin{array}{cc}
\Gamma^{*} \boldsymbol{Q} & \Gamma_{T}^{*} M \\
\Delta^{*} \boldsymbol{Q} & \Delta_{T}^{*} M
\end{array}\right]\left(\begin{array}{c}
f \\
f(T)
\end{array}\right),
$$

where the operator $W$ is defined in (2.3). By Lemma 2.1 the inverse $W^{-1}$ exists and is given by (2.5). Hence, there exists a unique strategy pair $(u, v)$ given by

$$
\left(\begin{array}{l}
u \\
v
\end{array}\right)=-W^{-1}\left[\begin{array}{cc}
\Gamma^{*} \boldsymbol{Q} & \Gamma_{T}^{*} M \\
\Delta^{*} \boldsymbol{Q} & \Delta_{T}^{*} M
\end{array}\right]\left(\begin{array}{c}
f \\
f(T)
\end{array}\right),
$$

which satisfies (2.14) and (2.15). Hence, this strategy pair is optimal. The existence and uniqueness of an optimal strategy pair is proved.

Finally, replacing the involved adjoint operators in (2.14) and (2.15) by their explicit forms given in (2.2), we can get (2.9) and (2.10). The backward argument also shows that if a specific strategy pair $(u, v)$ satisfies the relations (2.9) and (2.10), then the saddle point inequalities in (1.3) are satisfied, so that this strategy pair must be optimal. The proof of this theorem is completed.

\section{Projection causality}

In this section, we shall introduce the concepts related to the feature methodology of this work, which is called projection causality. Before doing so, we have a comment on the open-loop characterization (2.9) and (2.10) of the optimal strategy pair $(u, v)$. In these two expressions, the real-time values of strategy $u(t)$ and strategy $v(t)$ are determined in terms of the future-time values of the corresponding output $\{y(s ; u, v): t \leq s \leq T\}$. Therefore, these expressions are not a causal implementation of the optimal $(u, v)$.

The key issue of this work is to convert such an anti-causal dependence into a causal one. This task is very challenging because the kernels $G(\cdot, \cdot), K(\cdot, \cdot)$ and the data function $f(\cdot)$ in this Volterra integral system do not have the semigroup property, and the dynamical programming approach (which is effective in dealing with optimization problems for differential equations) will not help to achieve a causal feedback in this case. We need a new idea to attack this difficulty and some mathematical technicalities to accomplish the task of causal synthesis. The idea comes from a truncation projection defined as follows. 
Definition 3.1. Define a truncation operator $P_{\xi}$ on $\boldsymbol{U} \times \boldsymbol{V}$ by

$$
\left[P_{\xi}\left(\begin{array}{l}
u \\
v
\end{array}\right)\right](t)=\left(\begin{array}{l}
u(t) \\
v(t)
\end{array}\right) \text { for } t \in[0, \xi] ; \text { or }=\left(\begin{array}{l}
0 \\
0
\end{array}\right) \text { for } t \in(\xi, T],
$$

where $0 \leq \xi \leq T$ is a parameter which can be chosen arbitrarily.

It is obvious that $P_{\xi}$ and $I-P_{\xi}$ are both projections on the space $\boldsymbol{U} \times \boldsymbol{V}$. Moreover, $P_{\xi} \in L(\boldsymbol{U} \times \boldsymbol{V})$ can be expressed as $P_{\xi}=\operatorname{diag}\left(P_{\xi 1}, P_{\xi 2}\right)$ where $P_{\xi 1}$ and $P_{\xi 2}$ are respectively the truncation operators on $\boldsymbol{U}$ and on $\boldsymbol{V}$. We call these projections causal projections.

Note that $P_{\xi 1}$ (resp. $\left.P_{\xi 2}\right)$ commutes with $R_{1}\left(\right.$ resp. $\left.R_{2}\right)$ and $R_{1}^{-1}$ (resp. $R_{2}^{-1}$ ).

Denote by

$$
\boldsymbol{U}_{\xi}=\left(I-P_{\xi 1}\right) \boldsymbol{U}, \boldsymbol{V}_{\xi}=\left(I-P_{\xi 1}\right) \boldsymbol{V}, \text { and } \boldsymbol{U}_{\xi} \times \boldsymbol{V}_{\xi}=\left(I-P_{\xi}\right)(\boldsymbol{U} \times \boldsymbol{V})
$$

These subspaces are isomorphic to $L^{2}(\xi, T ; U), L^{2}(\xi, T ; V)$, and $L^{2}(\xi, T ; U \times V)$ respectively. Define a parametrized operator $W_{\xi}$ by

$$
W_{\xi}=\left.\left(I-P_{\xi}\right) W\right|_{\left(\boldsymbol{U}_{\xi} \times \boldsymbol{V}_{\xi}\right)},
$$

which is the restriction of $\left(I-P_{\xi}\right) W$ on the subspace $\boldsymbol{U}_{\xi} \times \boldsymbol{V}_{\xi}$. The component operators in the expression of $W_{\xi}$, as the analogue of $W$ in (2.3), are denoted by $\Phi_{\xi}, S_{\xi}$, and $\Psi_{\xi}$.

Lemma 3.2. For any $\xi \in[0, T)$, the operator $W_{\xi} \in L\left(\boldsymbol{U}_{\xi} \times \boldsymbol{V}_{\xi}\right)$ is self-adjoint and invertible. There is a constant $C_{W}$ independent of $0 \leq \xi<t$, such that

$$
\left\|W_{\xi}^{-1}\right\| \leq C_{W}, \text { for } \xi \in[0, T) .
$$

Proof. Similar to (2.5), here we have the inverse operator (which can be verified):

$$
W_{\xi}^{-1}=\left[\begin{array}{cc}
A_{\xi}^{-1} & -A_{\xi}^{-1} S_{\xi} \Psi_{\xi}^{-1} \\
-B_{\xi}^{-1} S_{\xi}^{*} \Phi_{\xi}^{-1} & B_{\xi}^{-1}
\end{array}\right]
$$

where

$$
\begin{aligned}
& A_{\xi}=\Phi_{\xi}-\left(I-P_{\xi 1}\right) S_{\xi} \Psi_{\xi}^{-1} S_{\xi}^{*}\left(I-P_{\xi 1}\right), \\
& B_{\xi}=\Psi_{\xi}-\left(I-P_{\xi 2}\right) S_{\xi}^{*} \Phi_{\xi}^{-1} S_{\xi}\left(I-P_{\xi 2}\right) .
\end{aligned}
$$

Since there exists a constant $\varepsilon_{0}>0$ such that $A_{\xi} \geq \Phi_{\xi} \geq \varepsilon_{0} I$ on $\boldsymbol{U}_{\xi}$ and $B_{\xi} \leq$ $\Psi_{\xi} \leq-\varepsilon_{0} I$ on $V_{\xi}$, we have $\left\|A_{\xi}^{-1}\right\|,\left\|\Phi_{\xi}^{-1}\right\|,\left\|B_{\xi}^{-1}\right\|$, and $\left\|\Psi_{\xi}^{-1}\right\| \leq\left(\varepsilon_{0}\right)^{-1}$. Then by the expression (3.5), we can conclude that (3.4) holds.

Definition 3.3. For any output trajectory $y(t ; u, v)$, we define the $\xi$-causal trajectory $y_{\xi}(\cdot)$ by

$$
y_{\xi}(t)=f(t)+\int_{0}^{t}\left[G(t, s)\left(P_{\xi 1} u\right)(s)+K(t, s)\left(P_{\xi 2} v\right)(s)\right] d s, \quad t \in[0, T] .
$$

One can call $\xi$-causal trajectory $y_{\xi}$ a semicausal trajectory, because the data function $f$ is not truncated while two control functions $u$ and $v$ are truncated at the time instant $\xi$. This concept is crucial to the causal synthesis of this integral game problem for the following reasons: As we mentioned earlier, since the data function $f$ is not semigroup-like, it will be impossible to get a synthesis result which determines the real-time values of the optimal strategy $(u(t), v(t))$ in terms of an output feedback only involving the information of $\{y(\tau): 0 \leq \tau \leq t\}$. Otherwise, the segment $\{f(s): t<s \leq T\}$ would have no influence at all on the optimal process over the subinterval $[0, t]$, which is certainly wrong in general. 
This insight leads one to believe that the best synthesis one can hope for is a feedback determination of the real-time optimal strategy $(u(t), v(t))$ in terms of the modified output $y_{t}(\cdot)$, which is exactly the $t$-causal trajectory, because $y_{1}(\cdot)$ does not involve any future unknown information about $\{u, v\}$ beyond the real-time $t$ and does involve the full information about the data function $f$ over the whole interval $[0, T]$. Indeed, what follows is just to materialize this conjecture.

Note that by Definition 3.3, we have

$$
\begin{aligned}
y_{\xi} & =f+\Gamma P_{\xi 1} u+\Delta P_{\xi 2} v, \\
y & =y_{\xi}+\Gamma\left(I-P_{\xi 1}\right) u+\Delta\left(I-P_{\xi 2}\right) v, \\
y(T) & =y_{\xi}(T)+\Gamma_{T}\left(I-P_{\xi 1}\right) u+\Delta_{T}\left(I-P_{\xi 2}\right) v .
\end{aligned}
$$

The next definition will provide an abstract evolutionary operator in the $\xi$-causality sense.

Definition 3.4. For any $\xi \in[0, T]$, define a $\xi$-evolutionary operator $E_{\xi} \in L(\boldsymbol{Y} \times Y)$ by

$$
E_{\xi}=I_{\boldsymbol{U} \times \boldsymbol{V}}-\left[\begin{array}{cc}
\Gamma & \Delta \\
\Gamma_{T} & \Delta_{T}
\end{array}\right] W_{\xi}^{-1}\left(I-P_{\xi}\right)\left[\begin{array}{cc}
\Gamma^{*} \boldsymbol{Q} & \Gamma_{T}^{*} M \\
\Delta^{*} \boldsymbol{Q} & \Delta_{T}^{*} M
\end{array}\right]
$$

Now we prove two important instrumental identities which will be used many times later on in establishing the synthesis result.

Lemma 3.5. The following identities hold,

$$
W_{\xi}^{-1}\left(I-P_{\xi}\right)\left[\begin{array}{cc}
\Gamma^{*} \boldsymbol{Q} & \Gamma_{T}^{*} M \\
\Delta^{*} \boldsymbol{Q} & \Delta_{T}^{*} M
\end{array}\right]=\left(I-P_{\xi}\right)\left[\begin{array}{cc}
R_{1}^{-1} & 0 \\
0 & R_{2}^{-1}
\end{array}\right]\left[\begin{array}{cc}
\Gamma^{*} \boldsymbol{Q} & \Gamma_{T}^{*} M \\
\Delta^{*} \boldsymbol{Q} & \Delta_{T}^{*} M
\end{array}\right] E_{\xi}
$$

and

$$
\left[\begin{array}{cc}
\Gamma & \Delta \\
\Gamma_{T} & \Delta_{T}
\end{array}\right] W_{\xi}^{-1}\left(I-P_{\xi}\right)=E_{\xi}\left[\begin{array}{cc}
\Gamma & \Delta \\
\Gamma_{T} & \Delta_{T}
\end{array}\right]\left(I-P_{\xi}\right)\left[\begin{array}{cc}
R_{1}^{-1} & 0 \\
0 & R_{2}^{-1}
\end{array}\right]
$$

where the two sides of (3.10) are operators from $\boldsymbol{Y} \times Y$ to $\boldsymbol{U}_{\xi} \times \boldsymbol{V}_{\xi}$, and the two sides of (3.11) are operators from $\boldsymbol{U} \times \boldsymbol{V}$ to $\boldsymbol{Y} \times Y$.

Proof. The verification of the two identities is straightforward by using the idempotent property of the projection $I-P_{\xi}$, the commutative property

$$
\left(I-P_{\xi}\right)\left[\begin{array}{cc}
R_{1}^{-1} & 0 \\
0 & R_{2}^{-1}
\end{array}\right]=\left[\begin{array}{cc}
R_{1}^{-1} & 0 \\
0 & R_{2}^{-1}
\end{array}\right]\left(I-P_{\xi}\right)
$$

and the following property which comes from (2.3),

$$
W-\left[\begin{array}{cc}
R_{1} & 0 \\
0 & R_{2}
\end{array}\right]=\left[\begin{array}{cc}
\Gamma^{*} \boldsymbol{Q} & \Gamma_{T}^{*} M \\
\Delta^{*} \boldsymbol{Q} & \Delta_{T}^{*} M
\end{array}\right]\left[\begin{array}{cc}
\Gamma & \Delta \\
\Gamma_{T} & \Delta_{T}
\end{array}\right]
$$


In detail, (3.10) follows from

$$
\begin{aligned}
& \left(I-P_{\xi}\right)\left[\begin{array}{cc}
R_{1}^{-1} & 0 \\
0 & R_{2}^{-1}
\end{array}\right]\left[\begin{array}{cc}
\Gamma^{*} \boldsymbol{Q} & \Gamma_{T}^{*} M \\
\Delta^{*} \boldsymbol{Q} & \Delta_{T}^{*} M
\end{array}\right] E_{\xi} \\
& =\left(I-P_{\xi}\right)\left[\begin{array}{cc}
R_{1}^{-1} & 0 \\
0 & R_{2}^{-1}
\end{array}\right]\left[\begin{array}{cc}
\Gamma^{*} \boldsymbol{Q} & \Gamma_{T}^{*} M \\
\Delta^{*} \boldsymbol{Q} & \Delta_{T}^{*} M
\end{array}\right] I_{\boldsymbol{U} \times \boldsymbol{V}} \\
& -\left(I-P_{\xi}\right)\left[\begin{array}{cc}
R_{1}^{-1} & 0 \\
0 & R_{2}^{-1}
\end{array}\right]\left[\begin{array}{cc}
\Gamma^{*} \boldsymbol{Q} & \Gamma_{T}^{*} M \\
\Delta^{*} \boldsymbol{Q} & \Delta_{T}^{*} M
\end{array}\right]\left[\begin{array}{cc}
\Gamma & \Delta \\
\Gamma_{T} & \Delta_{T}
\end{array}\right] \\
& \cdot W_{\xi}^{-1}\left(I-P_{\xi}\right)\left[\begin{array}{cc}
\Gamma^{*} \boldsymbol{Q} & \Gamma_{T}^{*} M \\
\Delta^{*} \boldsymbol{Q} & \Delta_{T}^{*} M
\end{array}\right] \\
& =\left[\begin{array}{cc}
R_{1}^{-1} & 0 \\
0 & R_{2}^{-1}
\end{array}\right]\left\{I-\left(I-P_{\xi}\right)\left[\begin{array}{cc}
\Gamma^{*} \boldsymbol{Q} & \Gamma_{T}^{*} M \\
\Delta^{*} \boldsymbol{Q} & \Delta_{T}^{*} M
\end{array}\right]\left[\begin{array}{cc}
\Gamma & \Delta \\
\Gamma_{T} & \Delta_{T}
\end{array}\right] W_{\xi}^{-1}\right\} \\
& \cdot\left(I-P_{\xi}\right)\left[\begin{array}{cc}
\Gamma^{*} \boldsymbol{Q} & \Gamma_{T}^{*} M \\
\Delta^{*} \boldsymbol{Q} & \Delta_{T}^{*} M
\end{array}\right] \\
& =\left[\begin{array}{cc}
R_{1}^{-1} & 0 \\
0 & R_{2}^{-1}
\end{array}\right]\left\{I-\left(I-P_{\xi}\right)\left(W-\left[\begin{array}{cc}
R_{1} & 0 \\
0 & R_{2}
\end{array}\right]\right) W_{\xi}^{-1}\right\} \\
& \cdot\left(I-P_{\xi}\right)\left[\begin{array}{cc}
\Gamma^{*} \boldsymbol{Q} & \Gamma_{T}^{*} M \\
\Delta^{*} \boldsymbol{Q} & \Delta_{T}^{*} M
\end{array}\right] \\
& =\left[\begin{array}{cc}
R_{1}^{-1} & 0 \\
0 & R_{2}^{-1}
\end{array}\right]\left(I-P_{\xi}\right)\left[\begin{array}{cc}
R_{1} & 0 \\
0 & R_{2}
\end{array}\right] W_{\xi}^{-1}\left(I-P_{\xi}\right)\left[\begin{array}{cc}
\Gamma^{*} \boldsymbol{Q} & \Gamma_{T}^{*} M \\
\Delta^{*} \boldsymbol{Q} & \Delta_{T}^{*} M
\end{array}\right] \\
& =W_{\xi}^{-1}\left(I-P_{\xi}\right)\left[\begin{array}{cc}
\Gamma^{*} \boldsymbol{Q} & \Gamma_{T}^{*} M \\
\Delta^{*} \boldsymbol{Q} & \Delta_{T}^{*} M
\end{array}\right] .
\end{aligned}
$$

The identity (3.11) follows from

$$
\begin{aligned}
& E_{\xi}\left[\begin{array}{cc}
\Gamma & \Delta \\
\Gamma_{T} & \Delta_{T}
\end{array}\right]\left(I-P_{\xi}\right)\left[\begin{array}{cc}
R_{1}^{-1} & 0 \\
0 & R_{2}^{-1}
\end{array}\right] \\
&=\left[\begin{array}{cc}
\Gamma & \Delta \\
\Gamma_{T} & \Delta_{T}
\end{array}\right]\left(I-P_{\xi}\right)\left[\begin{array}{cc}
R_{1}^{-1} & 0 \\
0 & R_{2}^{-1}
\end{array}\right] \\
&-\left[\begin{array}{cc}
\Gamma & \Delta \\
\Gamma_{T} & \Delta_{T}
\end{array}\right] W_{\xi}^{-1}\left(I-P_{\xi}\right)\left[\begin{array}{cc}
\Gamma^{*} \boldsymbol{Q} & \Gamma_{T}^{*} M \\
\Delta^{*} \boldsymbol{Q} & \Delta_{T}^{*} M
\end{array}\right]\left[\begin{array}{cc}
\Gamma & \Delta \\
\Gamma_{T} & \Delta_{T}
\end{array}\right] \\
& \cdot\left(I-P_{\xi}\right)\left[\begin{array}{cc}
R_{1}^{-1} & 0 \\
0 & R_{2}^{-1}
\end{array}\right] \\
&= {\left[\begin{array}{cc}
\Gamma & \Delta \\
\Gamma_{T} & \Delta_{T}
\end{array}\right]\left\{I-W_{\xi}^{-1}\left(I-P_{\xi}\right)\left[\begin{array}{cc}
\Gamma^{*} \boldsymbol{Q} & \Gamma_{T}^{*} M \\
\Delta^{*} Q & \Delta_{T}^{*} M
\end{array}\right]\left[\begin{array}{cc}
\Gamma & \Delta \\
\Gamma_{T} & \Delta_{T}
\end{array}\right]\right\} } \\
& \cdot\left[\begin{array}{cc}
R_{1}^{-1} & 0 \\
0 & R_{2}^{-1}
\end{array}\right]\left(I-P_{\xi}\right) \\
&= {\left[\begin{array}{cc}
\Gamma & \Delta \\
\Gamma_{T} & \Delta_{T}
\end{array}\right]\left\{I-W_{\xi}^{-1}\left(I-P_{\xi}\right)\left(W-\left[\begin{array}{cc}
R_{1} & 0 \\
0 & R_{2}
\end{array}\right]\right)\right\}\left[\begin{array}{cc}
R_{1}^{-1} & 0 \\
0 & R_{2}^{-1}
\end{array}\right]\left(I-P_{\xi}\right) } \\
&= {\left[\begin{array}{cc}
\Gamma & \Delta \\
\Gamma_{T} & \Delta_{T}
\end{array}\right] W_{\xi}^{-1}\left(I-P_{\xi}\right) . }
\end{aligned}
$$

The proof is completed. 


\section{CAusal Synthesis PRinciple}

In this section, we shall study the interrelationship among the parametrized operator $W_{\xi}$, the $\xi$-evolutionary operator $E_{\xi}$, and the $\xi$-causal trajectory $y_{\xi}(\cdot)$. Then we shall prove the causal synthesis result about the optimal strategy at the level that the synthesis expression is in terms of the abstract operators on the fibre spaces. Such an intermediate result gives rise to the abstract synthesis principle for this type of integral game problems. We call it the causal synthesis principle.

The first result in this section clarifies the relation between the $\xi$-evolutionary operator $E_{\xi}$ and the $\xi$-causal output trajectory $y_{\xi}$. It also justifies the reason why we call $E_{\xi}$ the $\xi$-evolutionary operator.

Theorem 4.1 (The evolution of optimality). Let $x \in[0, T]$ be arbitrarily given. The optimal output trajectory $y(\cdot)$ and the corresponding $\xi$-causal trajectory $y_{\xi}(\cdot)$ are related by

$$
\left(\begin{array}{c}
y \\
y(T)
\end{array}\right)=E_{\xi}\left(\begin{array}{c}
y_{\xi} \\
y_{\xi}(T)
\end{array}\right) \text { in } \boldsymbol{Y} \times Y
$$

Proof. From (2.14) and (2.15), which are satisfied by the optimal strategy $(u, v)$, together with (3.8), it follows that $\left(I-P_{\xi}\right)\left(\begin{array}{l}u \\ v\end{array}\right)$ satisfies the following equation,

$$
\begin{aligned}
R_{1}\left(I-P_{\xi 1}\right) u & +\Gamma^{*} \boldsymbol{Q} \Gamma\left(I-P_{\xi 1}\right) u+\Gamma_{T}^{*} M \Gamma_{T}\left(I-P_{\xi 1}\right) u \\
& +\Gamma^{*} \boldsymbol{Q} \Delta\left(I-P_{\xi 2}\right) v+\Gamma_{T}^{*} M \Delta_{T}\left(I-P_{\xi 2}\right) v \\
=-\left[\Gamma^{*} \boldsymbol{Q} y_{\xi}\right. & \left.+\Gamma_{T}^{*} M y_{\xi}(T)\right],
\end{aligned}
$$

and

$$
\begin{gathered}
R_{2}\left(I-P_{\xi 2}\right) v+\Delta^{*} \boldsymbol{Q} \Delta\left(I-P_{\xi 2}\right) v+\Delta_{T}^{*} M \Delta_{T}\left(I-P_{\xi 2}\right) v \\
+\Delta^{*} \boldsymbol{Q} \Gamma\left(I-P_{\xi 1}\right) u+\Delta_{T}^{*} M \Gamma_{T}\left(I-P_{\xi 1}\right) u \\
=-\left[\Delta^{*} \boldsymbol{Q} y_{\xi}+\Delta_{T}^{*} M y_{\xi}(T)\right] .
\end{gathered}
$$

By (3.13), we can write (4.2) and (4.3) together as one equation:

$$
W\left(I-P_{\xi}\right)\left(\begin{array}{c}
u \\
v
\end{array}\right)=-\left[\begin{array}{cc}
\Gamma^{*} \boldsymbol{Q} & \Gamma_{T}^{*} M \\
\Delta^{*} \boldsymbol{Q} & \Delta_{T}^{*} M
\end{array}\right]\left(\begin{array}{c}
y_{\xi} \\
y_{\xi}(T)
\end{array}\right) .
$$

Multiply two sides of (4.4) by $I-P_{\xi}$ from left, by (3.3), we obtain

$$
W_{\xi}\left(I-P_{\xi}\right)\left(\begin{array}{l}
u \\
v
\end{array}\right)=-\left(I-P_{\xi}\right)\left[\begin{array}{cc}
\Gamma^{*} \boldsymbol{Q} & \Gamma_{T}^{*} M \\
\Delta^{*} \boldsymbol{Q} & \Delta_{T}^{*} M
\end{array}\right]\left(\begin{array}{c}
y_{\xi} \\
y_{\xi}(T)
\end{array}\right)
$$

then by Lemma 3.2,

$$
\left(I-P_{\xi}\right)\left(\begin{array}{c}
u \\
v
\end{array}\right)=-W_{\xi}^{-1}\left(I-P_{\xi}\right)\left[\begin{array}{cc}
\Gamma^{*} \boldsymbol{Q} & \Gamma_{T}^{*} M \\
\Delta^{*} \boldsymbol{Q} & \Delta_{T}^{*} M
\end{array}\right]\left(\begin{array}{c}
y_{\xi} \\
y_{\xi}(T)
\end{array}\right)
$$

Since from (3.8) we have

$$
\left(\begin{array}{c}
y \\
y(T)
\end{array}\right)=\left(\begin{array}{c}
y_{\xi} \\
y_{\xi}(T)
\end{array}\right)+\left[\begin{array}{cc}
\Gamma & \Delta \\
\Gamma_{T} & \Delta_{T}
\end{array}\right]\left(I-P_{\xi}\right)\left(\begin{array}{l}
u \\
v
\end{array}\right)
$$


a substitution of (4.6) into the right-hand side of (4.7) gives us

$$
\begin{aligned}
\left(\begin{array}{c}
y \\
y(T)
\end{array}\right) & =\left\{I-\left[\begin{array}{cc}
\Gamma & \Delta \\
\Gamma_{T} & \Delta_{T}
\end{array}\right]\left(I-P_{\xi}\right) W_{\xi}^{-1}\left(I-P_{\xi}\right)\left[\begin{array}{cc}
\Gamma^{*} \boldsymbol{Q} & \Gamma_{T}^{*} M \\
\Delta^{*} \boldsymbol{Q} & \Delta_{T}^{*} M
\end{array}\right]\right\}\left(\begin{array}{c}
y_{\xi} \\
y_{\xi}(T)
\end{array}\right) \\
& =E_{\xi}\left(\begin{array}{c}
y_{\xi} \\
y_{\xi}(T)
\end{array}\right)
\end{aligned}
$$

which holds in $\boldsymbol{Y} \times Y$.

This theorem shows that the entire optimal output trajectory $y(\cdot)$, including its terminal value $y(T)$, is determined completely by the semicausal trajectory $y_{\xi}(\cdot)$ and its terminal value $y_{\xi}(T)$ for any chosen $\xi \in[0, T]$. This dependence is via the $\xi$ evolutionary operator $E_{\xi}$. Note that the $\xi$-causal trajectory and its terminal value depend only upon the optimal strategy pair $(u(t), v(t))$ during the time interval $0 \leq t \leq \xi$ and on the data function $f$. This result leads to the causal synthesis principle below.

Theorem 4.2 (Causal Synthesis Principle). A strategy pair $(u, v)$ is optimal if and only if

$$
\left(\begin{array}{l}
u(t) \\
v(t)
\end{array}\right)=-\left[\begin{array}{cc}
R_{1}^{-1} & 0 \\
0 & R_{2}^{-1}
\end{array}\right]\left[\begin{array}{cc}
\Gamma^{*}(t) \boldsymbol{Q} & \Gamma_{T}^{*}(t) M \\
\Delta^{*}(t) \boldsymbol{Q} & \Delta_{T}^{*}(t) M
\end{array}\right] E_{t}\left(\begin{array}{c}
y_{t} \\
y_{t}(T)
\end{array}\right), \quad t \in[0, T]
$$

where $y_{t}$ is the $t$-causal trajectory corresponding to this strategy pair $(u, v)$, and

$$
\begin{array}{lll}
\Gamma^{*}(t) \varphi=\left(\Gamma^{*} \varphi\right)(t), & \Delta^{*}(t) \varphi=\left(\Delta^{*} \varphi\right)(t), & \forall \varphi \in \boldsymbol{Y}, \\
\Gamma_{T}^{*}(t) z=\left(\Gamma_{T}^{*} z\right)(t), & \Delta_{T}^{*}(t) z=\left(\Delta_{T}^{*} z\right)(t), & \forall z \in Y .
\end{array}
$$

Proof. By Theorem 2.2, either (2.9)-(2.10) or (2.14)-(2.15), the optimal strategy pair $(u, v)$ must satisfy

$$
\left(\begin{array}{c}
u(t) \\
v(t)
\end{array}\right)=-\left[\begin{array}{cc}
R_{1}^{-1} & 0 \\
0 & R_{2}^{-1}
\end{array}\right]\left[\begin{array}{cc}
\Gamma^{*}(t) \boldsymbol{Q} & \Gamma_{T}^{*}(t) M \\
\Delta^{*}(t) \boldsymbol{Q} & \Delta_{T}^{*}(t) M
\end{array}\right]\left(\begin{array}{c}
y_{t} \\
y_{t}(T)
\end{array}\right), \quad t \in[0, T] .
$$

Substituting the relation (4.1) into (4.11), we obtain

$$
\left(\begin{array}{c}
u(t) \\
v(t)
\end{array}\right)=-\left[\begin{array}{cc}
R_{1}^{-1} & 0 \\
0 & R_{2}^{-1}
\end{array}\right]\left[\begin{array}{cc}
\Gamma^{*}(t) \boldsymbol{Q} & \Gamma_{T}^{*}(t) M \\
\Delta^{*}(t) \boldsymbol{Q} & \Delta_{T}^{*}(t) M
\end{array}\right] E_{\xi}\left(\begin{array}{c}
y_{\xi} \\
y_{\xi}(T)
\end{array}\right), \quad t \in[0, T],
$$

where $\xi \in[0, T]$ can be arbitrarily chosen. The choice $\xi=t$ then yields (4.9).

Conversely, in order to show that if $(u, v)$ satisfies (4.9) then it must be the optimal strategy pair, it suffices to prove that the pair $(u, v)$ satisfying (4.9) is unique. Since the system equation (1.1) and the equation (4.9) are linear, it is enough to show that when $f=0$, the homogeneous equation (4.9) of $(u, v)$ in the form of

$$
\left(\begin{array}{l}
u(t) \\
v(t)
\end{array}\right)=-\left[\begin{array}{cc}
R_{1}^{-1} & 0 \\
0 & R_{2}^{-1}
\end{array}\right]\left[\begin{array}{cc}
\Gamma^{*}(t) \boldsymbol{Q} & \Gamma_{T}^{*}(t) M \\
\Delta^{*}(t) \boldsymbol{Q} & \Delta_{T}^{*}(t) M
\end{array}\right] E_{t}\left[\begin{array}{cc}
\Gamma & \Delta \\
\Gamma_{T} & \Delta_{T}
\end{array}\right] P_{t}\left(\begin{array}{l}
u \\
v
\end{array}\right), \quad t \in[0, T]
$$

has only the zero solution. In fact, by Lemma 3.2 and (3.9) , we have

$$
\sup \left\{\left\|E_{\xi}\right\|_{L(\boldsymbol{Y} \times Y)}: \xi \in[0, T]\right\} \leq \text { const. }
$$


Moreover, from (2.2) and (4.10), we see that

$$
\sup \left\{\left\|\left[\begin{array}{cc}
\Gamma^{*}(t) \boldsymbol{Q} & \Gamma_{T}^{*}(t) M \\
\Delta^{*}(t) \boldsymbol{Q} & \Delta_{T}^{*}(t) M
\end{array}\right]\right\|_{L(\boldsymbol{Y} \times Y, U \times V)}: t \in[0, T]\right\} \leq \text { const. }
$$

Therefore, from (4.13) it follows that

$$
\begin{aligned}
\left\|\left(\begin{array}{l}
u(t) \\
v(t)
\end{array}\right)\right\|^{2} & \leq \text { const }\left\|P_{t}\left(\begin{array}{l}
u \\
v
\end{array}\right)\right\|_{L(\boldsymbol{U} \times \boldsymbol{V})}^{2} \\
& =\mathrm{const} \int_{0}^{t}\left\|\left(\begin{array}{l}
u(s) \\
v(s)
\end{array}\right)\right\|^{2} d s, \quad t \in[0, T] .
\end{aligned}
$$

By the Gronwall inequality, it follows that the solution $(u, v)$ of Eq. 4.13) must be $u(t)=0$ and $v(t)=0$ for $t \in[0, T]$. This completes the proof.

\section{Fredholm integral EQUATion For FeEdBack operator}

Based on the causal synthesis principle shown in Theorem 4.2, the real-time values of the optimal strategy pair $(u(t), v(t))$ are related to the $t$-causal output trajectory $y_{t}(\cdot)$ by three bounded linear operators, among which the operator $E_{t}$ is at a very abstract level and needs to be materialized. From its definition (3.9), $E_{t}$ essentially relates to the inverse operator of $W_{t}$. This observation hints that the realization of $E_{t}$ and the associated feedback operator in the causal synthesis may take place by a solution of a linear integral operator equation which represents the inversion of $W_{t}$ and the linked operations.

The purpose of this section is to sort out this issue by providing a linear integral operator equation of Fredholm type, whose solution will be directly used as the feedback operator in the final synthesis result.

Let $\xi \in[0, T]$. Define an operator-valued function $N_{\xi}(t, \sigma)$ by

$$
N_{\xi}(t, \sigma)=-\left[\begin{array}{cc}
R_{1}^{-1} & 0 \\
0 & R_{2}^{-1}
\end{array}\right]\left[\begin{array}{cc}
\Gamma^{*}(t) \boldsymbol{Q} & \Gamma_{T}^{*}(t) M \\
\Delta^{*}(t) \boldsymbol{Q} & \Delta_{T}^{*}(t) M
\end{array}\right] E_{\xi}\left[\begin{array}{cc}
G(\cdot, \sigma) & K(\cdot, \sigma) \\
G(T, \sigma) & K(T, \sigma)
\end{array}\right] .
$$

We see that $N_{\xi}(t, \sigma) \in L(U \times V)$, and by the convention made in [A2], which is well defined for $(t, \sigma) \in[0, T] \times[0, T]$.

Theorem 5.1. For any given $\xi \in[0, T]$, there exists a unique solution $N_{\xi}(t, \sigma)$ of the following Fredholm integral operator equation,

$$
N_{\xi}(t, \sigma)+\int_{\xi}^{T} \Pi(t, s) N_{\xi}(s, \sigma) d s=-\Pi(t, \sigma), \quad(t, \sigma) \in[0, T]^{2},
$$

where $\Pi(t, \sigma)$ is defined by

$$
\begin{aligned}
\Pi(t, \sigma)=\left[\begin{array}{cc}
R_{1}^{-1} & 0 \\
0 & R_{2}^{-1}
\end{array}\right]\{ & {\left[\begin{array}{ll}
G^{*}(T, t) M G(T, \sigma) & G^{*}(T, t) M K(T, \sigma) \\
K^{*}(T, t) M G(T, \sigma) & K^{*}(T, t) M K(T, \sigma)
\end{array}\right] } \\
& \left.+\int_{\max (t, \sigma)}^{T}\left[\begin{array}{ll}
G^{*}(s, t) Q G(s, \sigma) & G^{*}(s, t) Q K(s, \sigma) \\
K^{*}(s, t) Q G(s, \sigma) & K^{*}(s, t) Q K(s, \sigma)
\end{array}\right] d s\right\} .
\end{aligned}
$$


Moreover, the solution of Eq. (5.2) is given by (5.1), which is strongly continuous in $t \in[0, T]$ and strongly piecewise continuous in $\sigma \in[0, T]$, such that

$$
\sup \left\{\left\|N_{\xi}(t, \sigma)\right\|:(\xi, t, \sigma) \in[0, T]^{3}\right\} \leq \text { const. }
$$

Proof. First we show that $N_{\xi}(t, \sigma)$ given by (5.1) is really a solution of Eq. (5.2). This also justifies the same notation we used in (5.1) and (5.2). This fact can be verified directly, starting from (5.1):

$$
\begin{aligned}
& N_{\xi}(t, \sigma)=-\left[\begin{array}{cc}
R_{1}^{-1} & 0 \\
0 & R_{2}^{-1}
\end{array}\right]\left[\begin{array}{cc}
\Gamma^{*}(t) \boldsymbol{Q} & \Gamma_{T}^{*}(t) M \\
\Delta^{*}(t) \boldsymbol{Q} & \Delta_{T}^{*}(t) M
\end{array}\right] E_{\xi}\left[\begin{array}{cc}
G(\cdot, \sigma) & K(\cdot, \sigma) \\
G(T, \sigma) & K(T, \sigma)
\end{array}\right] \\
& {[\text { by }(3.9)]=-\left[\begin{array}{cc}
R_{1}^{-1} & 0 \\
0 & R_{2}^{-1}
\end{array}\right]\left[\begin{array}{cc}
\Gamma^{*}(t) \boldsymbol{Q} & \Gamma_{T}^{*}(t) M \\
\Delta^{*}(t) \boldsymbol{Q} & \Delta_{T}^{*}(t) M
\end{array}\right]\left\{I_{\boldsymbol{U} \times \boldsymbol{V}}\right.} \\
& \left.-\left[\begin{array}{cc}
\Gamma & \Delta \\
\Gamma_{T} & \Delta_{T}
\end{array}\right] W_{\xi}^{-1}\left(I-P_{\xi}\right)\left[\begin{array}{cc}
\Gamma^{*} \boldsymbol{Q} & \Gamma_{T}^{*} M \\
\Delta^{*} \boldsymbol{Q} & \Delta_{T}^{*} M
\end{array}\right]\right\}\left[\begin{array}{cc}
G(\cdot, \sigma) & K(\cdot, \sigma) \\
G(T, \sigma) & K(T, \sigma)
\end{array}\right] \\
& {[\text { by }(3.10)]=-\left[\begin{array}{cc}
R_{1}^{-1} & 0 \\
0 & R_{2}^{-1}
\end{array}\right]\left[\begin{array}{cc}
\Gamma^{*}(t) \boldsymbol{Q} & \Gamma_{T}^{*}(t) M \\
\Delta^{*}(t) \boldsymbol{Q} & \Delta_{T}^{*}(t) M
\end{array}\right]\left[\begin{array}{cc}
G(\cdot, \sigma) & K(\cdot, \sigma) \\
G(T, \sigma) & K(T, \sigma)
\end{array}\right]} \\
& +\left[\begin{array}{cc}
R_{1}^{-1} & 0 \\
0 & R_{2}^{-1}
\end{array}\right]\left[\begin{array}{cc}
\Gamma^{*}(t) \boldsymbol{Q} & \Gamma_{T}^{*}(t) M \\
\Delta^{*}(t) \boldsymbol{Q} & \Delta_{T}^{*}(t) M
\end{array}\right]\left[\begin{array}{cc}
\Gamma & \Delta \\
\Gamma_{T} & \Delta_{T}
\end{array}\right]\left(I-P_{\xi}\right) \\
& \cdot\left[\begin{array}{cc}
R_{1}^{-1} & 0 \\
0 & R_{2}^{-1}
\end{array}\right]\left[\begin{array}{cc}
\Gamma^{*} \boldsymbol{Q} & \Gamma_{T}^{*} M \\
\Delta^{*} \boldsymbol{Q} & \Delta_{T}^{*} M
\end{array}\right] E_{\xi}\left[\begin{array}{cc}
G(\cdot, \sigma) & K(\cdot, \sigma) \\
G(T, \sigma) & K(T, \sigma)
\end{array}\right] \\
& {[\text { by }(5.1)]=-\left[\begin{array}{cc}
R_{1}^{-1} & 0 \\
0 & R_{2}^{-1}
\end{array}\right]\left[\begin{array}{cc}
\Gamma^{*}(t) \boldsymbol{Q} & \Gamma_{T}^{*}(t) M \\
\Delta^{*}(t) \boldsymbol{Q} & \Delta_{T}^{*}(t) M
\end{array}\right]\left[\begin{array}{cc}
G(\cdot, \sigma) & K(\cdot, \sigma) \\
G(T, \sigma) & K(T, \sigma)
\end{array}\right]} \\
& -\left[\begin{array}{cc}
R_{1}^{-1} & 0 \\
0 & R_{2}^{-1}
\end{array}\right]\left[\begin{array}{cc}
\Gamma^{*}(t) \boldsymbol{Q} & \Gamma_{T}^{*}(t) M \\
\Delta^{*}(t) \boldsymbol{Q} & \Delta_{T}^{*}(t) M
\end{array}\right]\left[\begin{array}{cc}
\Gamma & \Delta \\
\Gamma_{T} & \Delta_{T}
\end{array}\right]\left(I-P_{\xi}\right) N_{\xi}(\cdot, \sigma) \\
& \text { [by }(2.1),(2.2),(5.3)]=-\Pi(t, \sigma)-\int_{0}^{T} \Pi(t, s)\left[\left(I-P_{\xi}\right) N_{\xi}\right](s, \sigma) d s \\
& =-\Pi(t, \sigma)-\int_{\xi}^{T} \Pi(t, s) N_{\xi}(s, \sigma) d s .
\end{aligned}
$$

This shows that the operator function given in (5.1) is a solution of Eq. (5.2). From (5.1), it is easy to check that this solution $N_{\xi}(t, \sigma)$ satisfies the required continuity and uniform boundedness.

Now we can prove the uniqueness of solution for Eq. (5.2) by showing that for any $\xi \in[0, T]$, the corresponding homogeneous equation,

$$
N_{\xi}(t, \sigma)+\int_{\xi}^{T} \Pi(t, s) N_{\xi}(s, \sigma) d s=0,(t, \sigma) \in[0, T]^{2},
$$

admits only a trivial solution $N_{\xi}(t, \sigma)=0,(t, \sigma) \in[0, T]^{2}$. In fact, from (5.3), (2.1), and (2.2) we have

$$
\begin{aligned}
\int_{\xi}^{T} & \Pi(\cdot, s) N_{\xi}(s, \sigma) d s \\
\quad & =\left[\begin{array}{cc}
R_{1}^{-1} & 0 \\
0 & R_{2}^{-1}
\end{array}\right]\left[\begin{array}{cc}
\Gamma^{*} \boldsymbol{Q} \Gamma+\Gamma_{T}^{*} M \Gamma_{T} & \Gamma^{*} \boldsymbol{Q} \Delta+\Gamma_{T}^{*} M \Delta_{T} \\
\Delta^{*} \boldsymbol{Q} \Gamma+\Delta_{T}^{*} M \Gamma_{T} & \Delta^{*} \boldsymbol{Q} \Delta+\Delta_{T}^{*} M \Delta_{T}
\end{array}\right]\left(I-P_{\xi}\right) N_{\xi}(\cdot, \sigma) .
\end{aligned}
$$


Multiply the two sides of (5.6) by $\left(I-P_{\xi}\right)\left[\begin{array}{cc}R_{1} & 0 \\ 0 & R_{2}\end{array}\right]^{2}$, by (5.7) and (2.3) as well as the idempotent property of the projection $\left(I-P_{\xi}\right)$ and the commutative property (3.12), we obtain

$$
\left(I-P_{\xi}\right) W\left[\left(I-P_{\xi}\right) N_{\xi}(\cdot, \sigma)\right]=W_{\xi}\left[\left(I-P_{\xi}\right) N_{\xi}(\cdot, \sigma)\right]=0
$$

Then apply the inverse $W_{\xi}^{-1}$ to the equality (5.8), we get

$$
N_{\xi}(s, \sigma)=0, \forall(\xi, s, \sigma) \in[0, T] \times[\xi, T] \times[0, T]
$$

Substitute (5.9) into the integral on the left-hand side of Eq. (5.6), we finally obtain

$$
N_{\xi}(t, \sigma)=0, \forall(\xi, t, \sigma) \in[0, T] \times[0, T] \times[0, T] .
$$

Thus the proof is completed.

As an important remark, the kernel and the inhomogeneous term of the Fredholm integral equation (5.2) are independent of the data function $f$ and of the control functions $u$ and $v$. Therefore, in the computational implementation of the optimal strategies, one can solve this Fredholm integral equation in advance or separately by whatever numerical methods, and put the solution data in a storage ready for use.

The final result on the causal synthesis of the optimal strategy pair will be provided in the next section, in which this solution $N_{\xi}(t, \sigma)$ of Eq. (5.2) will be used as the feedback operator.

\section{Causal synthesis formula}

In this section, we shall present the final result on the causal synthesis of the optimal strategy pair for this quadratic integral game problem. Since this final result will eliminate any abstract operator on the fibre spaces, the implementation is a straightforward computation in the underlying Hilbert spaces, with the aid of the solution of Fredholm integral equation (5.2) only.

Lemma 6.1. A strategy pair $(u, v) \in \boldsymbol{U} \times \boldsymbol{V}$ is optimal if and only if it can be expressed by

$$
\begin{aligned}
\left(\begin{array}{c}
u(t) \\
v(t)
\end{array}\right)= & -\left(\begin{array}{c}
R_{1}^{-1}\left[\Gamma^{*}(t) \boldsymbol{Q} y_{t}+\Gamma_{T}^{*}(t) M y_{t}(T)\right] \\
R_{2}^{-1}\left[\Delta^{*}(t) \boldsymbol{Q} y_{t}+\Delta_{T}^{*}(t) M y_{t}(T)\right]
\end{array}\right) \\
& -\int_{t}^{T} N_{t}(t, \sigma)\left(\begin{array}{c}
R_{1}^{-1}\left[\Gamma^{*}(\sigma) \boldsymbol{Q} y_{t}+\Gamma_{T}^{*}(\sigma) M y_{t}(T)\right] \\
R_{2}^{-1}\left[\Delta^{*}(\sigma) \boldsymbol{Q} y_{t}+\Delta_{T}^{*}(\sigma) M y_{t}(T)\right]
\end{array}\right) d \sigma, \quad t \in[0, T]
\end{aligned}
$$

where $N_{\xi}(t, \sigma)$ is the unique solution of Eq. (5.2), and $y_{t}(\cdot)$ is the corresponding $t$-causal output trajectory, with $t$ being the real time. 
Proof. If $(u, v)$ is the optimal strategy pair whose corresponding output trajectory is $y$, then by Theorem 4.2 , we have

$$
\begin{aligned}
& \left(\begin{array}{c}
u(t) \\
v(t)
\end{array}\right)=-\left[\begin{array}{cc}
R_{1}^{-1} & 0 \\
0 & R_{2}^{-1}
\end{array}\right]\left[\begin{array}{cc}
\Gamma^{*}(t) \boldsymbol{Q} & \Gamma_{T}^{*}(t) M \\
\Delta^{*}(t) \boldsymbol{Q} & \Delta_{T}^{*}(t) M
\end{array}\right] E_{t}\left(\begin{array}{c}
y_{t} \\
y_{t}(T)
\end{array}\right) \\
& {[\text { by (3.9) }]=-\left[\begin{array}{cc}
R_{1}^{-1} & 0 \\
0 & R_{2}^{-1}
\end{array}\right]\left[\begin{array}{cc}
\Gamma^{*}(t) \boldsymbol{Q} & \Gamma_{T}^{*}(t) M \\
\Delta^{*}(t) \boldsymbol{Q} & \Delta_{T}^{*}(t) M
\end{array}\right]\left(\begin{array}{c}
y_{t} \\
y_{t}(T)
\end{array}\right)} \\
& -\left[\begin{array}{cc}
R_{1}^{-1} & 0 \\
0 & R_{2}^{-1}
\end{array}\right]\left[\begin{array}{cc}
\Gamma^{*}(t) \boldsymbol{Q} & \Gamma_{T}^{*}(t) M \\
\Delta^{*}(t) \boldsymbol{Q} & \Delta_{T}^{*}(t) M
\end{array}\right]\left[\begin{array}{cc}
\Gamma & \Delta \\
\Gamma_{T} & \Delta_{T}
\end{array}\right] \\
& \cdot W_{t}^{-1}\left(I-P_{t}\right)\left[\begin{array}{cc}
\Gamma^{*} \boldsymbol{Q} & \Gamma_{T}^{*} M \\
\Delta^{*} \boldsymbol{Q} & \Delta_{T}^{*} M
\end{array}\right]\left(\begin{array}{c}
y_{t} \\
y_{t}(T)
\end{array}\right) \\
& {[\text { by }(3.11)]=-\left[\begin{array}{cc}
R_{1}^{-1} & 0 \\
0 & R_{2}^{-1}
\end{array}\right]\left[\begin{array}{cc}
\Gamma^{*}(t) \boldsymbol{Q} & \Gamma_{T}^{*}(t) M \\
\Delta^{*}(t) \boldsymbol{Q} & \Delta_{T}^{*}(t) M
\end{array}\right]\left(\begin{array}{c}
y_{t} \\
y_{t}(T)
\end{array}\right)} \\
& -\left[\begin{array}{cc}
R_{1}^{-1} & 0 \\
0 & R_{2}^{-1}
\end{array}\right]\left[\begin{array}{cc}
\Gamma^{*}(t) \boldsymbol{Q} & \Gamma_{T}^{*}(t) M \\
\Delta^{*}(t) \boldsymbol{Q} & \Delta_{T}^{*}(t) M
\end{array}\right] E_{t}\left[\begin{array}{cc}
\Gamma & \Delta \\
\Gamma_{T} & \Delta_{T}
\end{array}\right]\left(I-P_{t}\right) \\
& \cdot\left[\begin{array}{cc}
R_{1}^{-1} & 0 \\
0 & R_{2}^{-1}
\end{array}\right]\left[\begin{array}{cc}
\Gamma^{*} \boldsymbol{Q} & \Gamma_{T}^{*} M \\
\Delta^{*} \boldsymbol{Q} & \Delta_{T}^{*} M
\end{array}\right]\left(\begin{array}{c}
y_{t} \\
y_{t}(T)
\end{array}\right) \\
& {[\text { by }(2.1),(2.2)]=-\left(\begin{array}{c}
R_{1}^{-1}\left[\Gamma^{*}(t) \boldsymbol{Q} y_{t}+\Gamma_{T}^{*}(t) M y_{t}(T)\right] \\
R_{2}^{-1}\left[\Delta^{*}(t) \boldsymbol{Q} y_{t}+\Delta_{T}^{*}(t) M y_{t}(T)\right]
\end{array}\right)} \\
& -\left[\begin{array}{cc}
R_{1}^{-1} & 0 \\
0 & R_{2}^{-1}
\end{array}\right]\left[\begin{array}{cc}
\Gamma^{*}(t) \boldsymbol{Q} & \Gamma_{T}^{*}(t) M \\
\Delta^{*}(t) \boldsymbol{Q} & \Delta_{T}^{*}(t) M
\end{array}\right] E_{t} \\
& \cdot \int_{t}^{T}\left[\begin{array}{cc}
G(\cdot, \sigma) & K(\cdot, \sigma) \\
G(T, \sigma) & K(T, \sigma)
\end{array}\right]\left(\begin{array}{c}
R_{1}^{-1}\left[\Gamma^{*}(\sigma) \boldsymbol{Q} y_{t}+\Gamma_{T}^{*}(\sigma) M y_{t}(T)\right] \\
R_{2}^{-1}\left[\Delta^{*}(\sigma) \boldsymbol{Q} y_{t}+\Delta_{T}^{*}(\sigma) M y_{t}(T)\right]
\end{array}\right) d \sigma \\
& {[\text { by }(5.1)]=-\left(\begin{array}{c}
R_{1}^{-1}\left[\Gamma^{*}(t) \boldsymbol{Q} y_{t}+\Gamma_{T}^{*}(t) M y_{t}(T)\right] \\
R_{2}^{-1}\left[\Delta^{*}(t) \boldsymbol{Q} y_{t}+\Delta_{T}^{*}(t) M y_{t}(T)\right]
\end{array}\right)} \\
& -\int_{t}^{T} N_{t}(t, \sigma)\left(\begin{array}{c}
R_{1}^{-1}\left[\Gamma^{*}(\sigma) \boldsymbol{Q} y_{t}+\Gamma_{T}^{*}(\sigma) M y_{t}(T)\right] \\
R_{2}^{-1}\left[\Delta^{*}(\sigma) \boldsymbol{Q} y_{t}+\Delta_{T}^{*}(\sigma) M y_{t}(T)\right]
\end{array}\right) d \sigma, \quad t \in[0, T] .
\end{aligned}
$$

This shows that the optimal strategy $(u, v)$ must satisfy the relation (6.1). Conversely, for any given data function $f$, there is only a unique game process $\{u, v, y\}$ which satisfies the relation (6.1). This can be proved in a similar way as in the proof of Theorem 5.1 by considering the solution of the homogeneous equation and using the Gronwall inequality. The detail of this argument is omitted.

Finally, we can present the main result of this paper, which provides the causal synthesis formula for the optimal strategy pair of this quadratic integral game problem.

Theorem 6.2 (Causal Synthesis Formula). Under the standing assumptions, a strategy pair $(u, v)$ is optimal if and only if it can be expressed by

$$
\left(\begin{array}{l}
u(t) \\
v(t)
\end{array}\right)=-H(T, t) M y_{t}(T)-\int_{t}^{T} H(s, t) Q y_{t}(s) d s, \quad t \in[0, T]
$$


where $y_{t}(\cdot)$ is the corresponding t-causal output trajectory and the feedback operator $H(s, t)$ is independently given by

$$
H(s, t)=\left(\begin{array}{l}
R_{1}^{-1} G^{*}(s, t) \\
R_{2}^{-1} K^{*}(s, t)
\end{array}\right)+\int_{t}^{s} N_{t}(t, \sigma)\left(\begin{array}{c}
R_{1}^{-1} G^{*}(s, \sigma) \\
R_{2}^{-1} K^{*}(s, \sigma)
\end{array}\right) d \sigma
$$

in which $N_{\xi}(t, \sigma)$ is the unique solution of the Fredholm integral operator equation (5.2).

Proof. Substitute (4.10) and (2.2) into (6.1), we obtain

$$
\begin{aligned}
\left(\begin{array}{c}
u(t) \\
v(t)
\end{array}\right)= & -\left(\begin{array}{l}
R_{1}^{-1} G^{*}(T, t) \\
R_{2}^{-1} K^{*}(T, t)
\end{array}\right) M y_{t}(T)-\int_{t}^{T}\left(\begin{array}{l}
R_{1}^{-1} G^{*}(s, t) \\
R_{2}^{-1} K^{*}(s, t)
\end{array}\right) Q y_{t}(s) d s \\
& -\int_{t}^{T} N_{t}(t, \sigma)\left(\begin{array}{c}
R_{1}^{-1} G^{*}(T, \sigma) \\
R_{2}^{-1} K^{*}(T, \sigma)
\end{array}\right) M y_{t}(T) d \sigma \\
& -\int_{t}^{T} N_{t}(t, \sigma) \int_{\sigma}^{T}\left(\begin{array}{l}
R_{1}^{-1} G^{*}(s, \sigma) \\
R_{2}^{-1} K^{*}(s, \sigma)
\end{array}\right) Q y_{t}(s) d s d \sigma .
\end{aligned}
$$

Interchanging the order of integration in the last term of (6.5), we can group together the first term and the third term on the right-hand side of 6.5 to get

$$
-H(T, t) M y_{t}(T)
$$

and group together the second term and the fourth term to get

$$
-\int_{t}^{T} H(s, t) Q y_{t}(s) d s
$$

where $H(s, t)$ is determined by (6.4). This shows that if $(u, v)$ is the optimal strategy pair, then it must satisfy the relation (6.3).

Conversely, if $(u, v)$ satisfies (6.3), then it satisfies (6.1) because (6.3) is no other than the concrete expression of (6.1) in terms of the original operators in the description of this game problem together with the gain operator $N_{t}(t, \sigma)$ coming from the solution of the Fredholm integral equation (5.2). By Lemma 6.1, such a strategy pair must be optimal. The proof is completed.

The following result is a corollary of Theorem 6.2, which provides an alternative scheme for the causal synthesis of the optimal strategy pair $(u, v)$. This scheme is to solve a Volterra integral equation and get the optimal strategy pair.

Theorem 6.3 (Causal Synthesis via Volterra Integral Equation). Under the standing assumptions, a strategy pair $(u, v)$ is optimal if and only if it satisfies the following linear Volterra integral equation,

$$
\begin{aligned}
\left(\begin{array}{l}
u(t) \\
v(t)
\end{array}\right)= & -H(T, t) M f(T)-\int_{t}^{T} H(s, t) Q f(s) d s \\
& -\int_{0}^{t} Z(t, \tau)\left(\begin{array}{l}
u(\tau) \\
v(\tau)
\end{array}\right) d \tau, \quad t \in[0, T]
\end{aligned}
$$


where $H(s, t)$ is given by (6.4), and the operator $Z(t, \tau)$ is given by

$$
\begin{aligned}
Z(t, \tau)= & {\left[\begin{array}{ll}
R_{1}^{-1} G^{*}(T, \tau) M G(T, \tau) & R_{1}^{-1} G^{*}(T, \tau) M K(T, \tau) \\
R_{2}^{-1} K^{*}(T, \tau) M G(T, \tau) & R_{2}^{-1} K^{*}(T, \tau) M K(T, \tau)
\end{array}\right] } \\
& +\int_{\tau}^{T} \int_{t}^{s} N_{t}(t, \sigma)\left[\begin{array}{ll}
R_{1}^{-1} G^{*}(s, \tau) Q G(s, \tau) & R_{1}^{-1} G^{*}(s, \tau) Q K(s, \tau) \\
R_{2}^{-1} K^{*}(s, \tau) Q G(s, \tau) & R_{2}^{-1} K^{*}(s, \tau) Q K(s, \tau)
\end{array}\right] d \sigma d s
\end{aligned}
$$

in which $N_{\xi}(t, \sigma)$ is the unique solution of the Fredholm integral operator equation (5.2).

Proof. By (3.7), we can substitute

$$
y_{t}(\tau)=f(\tau)+\int_{0}^{t}[G(\tau, s) u(s)+K(\tau, s) v(s)] d s, \tau \in[0, T]
$$

into the synthesis formula (6.3). Due to the convention made in [A2] that $G(t, s)=0$ and $K(t, s)=0$ whenever $(t, s) \in \mathbb{R}^{2} \backslash \Omega$, we can interchange the order for the double and triple integrals in that expression. Finally, we get (6.6) which is satisfied by the optimal strategy pair $(u, v)$ with kernel $Z(t, \tau)$ defined by (6.7).

Conversely, it is easy to show that the solution of Volterra integral equation (6.6) is unique by the Gronwall inequality. Thus any strategy $(u, v)$ satisfying Eq. (6.6) must be optimal. The proof is finished.

Remark 6.4. The main results achieved in this work are Theorem 6.2 (Causal Synthesis Formula) and Theorem6.3 (Causal Synthesis via Volterra Integral Equation). We emphasize that in each of these two causal syntheses, the feedback operators $H(s, t)$ or $Z(t, \tau)$ can be determined independently of the data function and player controls $\{f, u, v\}$. Precisely,

i) $H(s, t)$ is determined by (6.4),

ii) $Z(t, \tau)$ is determined by 6.7 ,

where in (6.4) and (6.7) the only thing we need to know is the operator function $N_{t}(t, \sigma)$ which is obtained from the unique solution of the Fredholm integral equation (5.2). This Fredholm integral equation plays a role similar to that of the operator Riccati equation in the linear-quadratic differential game problems.

In applications, the solution $N_{t}(t, \sigma)$ of Eq. (5.2) together with the feedback operators $H(s, t)$ and $Z(t, \tau)$ can be numerically acquired and stored separately and in advance. Then the implementation of the integral game by the synthesis procedure will be achieved either by using the formula (6.3) or by solving Volterra integral equation (6.6).

As we have pointed out earlier, for this general Volterra integral system (1.1) with data $f(\cdot)$ and kernels $G(\cdot, \cdot), K(\cdot, \cdot)$ not possessing the semigroup property, it is indeed impossible to implement the optimal strategy pair by the feedback of the actual trajectory $\{y(\tau): 0 \leq \tau \leq t\}$ because the future information of $f, G$, and $K$ must also be counted. So the obtained causal syntheses shown in Theorem 6.2 and Theorem 6.3 are the best synthesis results one can hope for in this general setting.

Remark 6.5. If one wishes to generalize these causal synthesis results toward the cutting edge, the assumption [A2] on the strong continuity of the two kernels $G(\cdot, \cdot)$ and $K(\cdot, \cdot)$ can be weakened. They may even have some singularity such as

$$
\|G(t, s)\|,\|K(t, s)\| \leq \mathrm{const} \frac{1}{|t-s|^{\alpha}}, \quad \text { for } 0 \leq s<t \leq T,
$$


where the exponent $0 \leq \alpha<1$ has certain restrictions. However, it is not the intention of this paper to explore those sharpening results in several possible directions. Further generalizations and numerical schemes are left for future investigations.

In the following two sections, we shall present two application examples which will illustrate the obtained causal synthesis, especially the causal synthesis formula presented in Theorem 6.2

The first example is a quadratic differential game of general linear delay systems. The second example is the standard linear quadratic differential game for which we shall specifically prove that, in this particular case, the new result eventually reduces to the known synthesis result in terms of the solution of a differential Riccati operator equation.

\section{Quadratic Differential game of Linear Delay Systems}

As the first example, we shall apply the obtained causal synthesis result to a quadratic differential game of a linear system with incommensurable and finite time delays in state variable and in two players' controls.

Let $Y, U$, and $V$ be real Hilbert spaces, which are respectively the valued spaces of the state $y(t)$, the players' controls $u(t)$ and $v(t)$, for $t \in[0, T]$. $T>0$ is finite and fixed. Suppose $0<h_{1}<\cdots<h_{n}, 0<r_{1}<\cdots<r_{m}$, and $0<\tau_{1}<\cdots<\tau_{p}$. Denote by $h=h_{n}, r=r_{m}$, and $\tau=\tau_{p}$. Assume that $\max \{h, r, \tau\} \ll T$.

Let $X=Y \times L^{2}(-h, 0 ; Y) \times L^{2}(-r, 0 ; U) \times L^{2}(-\tau, 0 ; V)$. Consider a linear evolution system with time delays,

$$
\begin{aligned}
\frac{d y(t)}{d t}= & A y(t)+\sum_{i=1}^{n} A_{i} y\left(t-h_{i}\right)+B u(t) \\
& +\sum_{j=1}^{m} B_{j} u\left(t-r_{j}\right)+C v(t)+\sum_{k=1}^{p} C_{k} v\left(t-\tau_{k}\right),
\end{aligned}
$$

with initial data given by

$$
\begin{gathered}
y(0)=y_{0} \\
x(\theta)=\varphi(\theta), \quad-h \leq \theta<0 \\
u(\xi)=\lambda(\xi), \quad-r \leq \xi \leq 0 \\
v(\zeta)=\psi(\zeta), \quad-\tau \leq \zeta \leq 0,
\end{gathered}
$$

where $\left\{y_{0}, \varphi, \lambda, \psi\right\} \in X$ is arbitrarily given. Assume that $A: D(A)(\subset Y) \rightarrow Y$ is an infinitesimal generator of a $C_{0}$-semigroup $e^{A t}(t \geq 0)$, and $A_{i} \in L(Y), i=1, \ldots, n, B$ and $B_{j} \in L(U, Y), j=1, \ldots, m, C$ and $C_{k} \in L(V, Y), k=1, \ldots, p$.

The state function is defined to be the mild solution of Eq. (7.1) in the usual sense, which satisfies the initial condition given in (7.2). Set up a quadratic criterion exactly in the previous form (1.2), with the assumption [A3] satisfied. We now consider the differential game problem of this delayed linear system (7.1) with respect to the performance functional (1.2) in the same saddle point sense described in (1.3).

First we shall formulate the input $(u, v)$ and output $y$ relation determined by Eq. (7.1) into a Volterra integral equation shown as (1.1), then we shall be able to apply our result to solve the synthesis problem of this differential game. The idea in handling this first step is using the fundamental solution $F(t):[0, \infty) \rightarrow L(Y)$ 
of the homogeneous equation corresponding to (7.1). Let $F(t)$ be the mild solution of the following operator equation

$$
\begin{gathered}
\frac{d F(t)}{d t}=A F(t)+\sum_{i=1}^{n} A_{i} F\left(t-h_{i}\right), \quad \text { for } t \geq 0, \\
F(0)=I(\text { identity on } Y) \\
F(t)=0, \quad \text { for } t<0 .
\end{gathered}
$$

This solution $F(t)$ is the fundamental solution associated with Eq. (7.1). We know that this fundamental solution exists uniquely and is given by

$$
\begin{gathered}
F(t)=e^{A t}+\int_{0}^{t} e^{A(t-s)} \sum_{i=1}^{n} A_{i} F\left(s-h_{i}\right) d s, \quad \text { for } t \geq 0 \\
=0, \quad \text { for } t<0 .
\end{gathered}
$$

This fundamental solution does not have the semigroup property. Instead, similar to the proof of the corresponding lemma in [5], we can prove that $F(t)$ satisfies the following evolutionary formula. Its proof is omitted.

Lemma 7.1. The fundamental solution $F(t)$ satisfies the following relation,

$$
F(t+s)=F(t) F(s)+\sum_{i=1}^{n} \int_{0}^{\min \left(t, h_{i}\right)} F(t-\sigma) A_{i} F\left(s+\sigma-h_{i}\right) d \sigma, t, s \geq 0
$$

In order to simplify the determination of specific integral limits, let us make a convention that

$$
\varphi(t)=0, \quad \lambda(t)=0, \quad \psi(t)=0, \quad \text { whenever } t>0 .
$$

Then the state function $y(t)$ can be expressed by

$$
y(t)=f(t)+\int_{0}^{t} G(t-s) u(s) d s+\int_{0}^{t} K(t-s) v(s) d s, \quad t \in[0, T],
$$

where

$$
\begin{aligned}
f(t) & =f\left(t ; y_{0}, \varphi, \lambda, \psi\right) \\
& =F(t) x_{0}+\int_{0}^{t} F(t-s)\left[\sum_{i=1}^{n} A_{i} \varphi\left(s-h_{i}\right)+\sum_{j=1}^{m} B_{j} \lambda\left(s-r_{j}\right)\right. \\
& \left.+\sum_{i=1}^{p} C_{i} \psi\left(s-\tau_{k}\right)\right] d s
\end{aligned}
$$

and

$$
\begin{gathered}
G(t)=F(t) B+F\left(t-r_{1}\right) B_{1}+\cdots+F\left(t-r_{m}\right) B_{m}, \quad t \geq 0, \\
K(t)=F(t) C+F\left(t-\tau_{1}\right) C_{1}+\cdots+F\left(t-\tau_{p}\right) C_{p}, \quad t \geq 0 .
\end{gathered}
$$

Therefore, the original differential game problem is reduced to an integral game problem described by the input-output equation (7.7) and the quadratic performance functional (1.2) in the saddle point sense (1.3). 
Lemma 7.2. $F(\cdot), G(\cdot)$ and $K(\cdot)$ satisfy the following relations,

$$
G(t+s)=F(t) G(s)+\sum_{i=1}^{n} \int_{0}^{\min \left(t, h_{i}\right)} F(t-\sigma) A_{i} G\left(s+\sigma-h_{i}\right) d \sigma, \quad t, s \geq 0,
$$

$$
K(t+s)=F(t) K(s)+\sum_{i=1}^{n} \int_{0}^{\min \left(t, h_{i}\right)} F(t-\sigma) A_{i} K\left(s+\sigma-h_{i}\right) d \sigma, \quad t, s \geq 0 .
$$

Proof. The two equalities can be verified directly by using (7.5), (7.9) and (7.10). The detail is omitted.

Lemma 7.3. For any initial data $\left(y_{0}, \varphi, \lambda, \psi\right) \in X$ and any admissible strategy pair $(u, v) \in L^{2}(0, T ; U) \times L^{2}(0, T ; V)$, the $\xi$-causal trajectory $y_{\xi}(t), \xi \in[0, T]$, satisfies the following relations,

$$
\begin{aligned}
y_{\xi}(t)= & y(t), \quad \text { for } t \leq \xi ; \\
y_{\xi}(t)= & F(t-\xi) y(\xi)+\sum_{i=1}^{n} \int_{\xi}^{\min \left(t, \xi+h_{i}\right)} F(t-\eta) A_{i} y\left(\eta-h_{i}\right) d \eta \\
& +\sum_{i=1}^{n} \int_{\min \left(\xi, h_{i}\right)}^{h_{i}} F(t-\eta) A_{i} \varphi\left(\eta-h_{i}\right) d \eta \\
& +\sum_{j=1}^{m} \int_{\min \left(\xi, r_{j}\right)}^{r_{j}} F(t-\eta) B_{i} \lambda\left(\eta-h_{i}\right) d \eta \\
& +\sum_{k=1}^{p} \int_{\min \left(\xi, \tau_{k}\right)}^{\tau_{k}} F(t-\eta) C_{i} \psi\left(\eta-h_{i}\right) d \eta, \quad \text { for } t \in(\xi, T] .
\end{aligned}
$$

Proof. The expression for $t>\xi$ can be verified by a straightforward but tedious calculation using the relations (7.7)-(7.9) and the properties (7.5), (7.11), and (7.12). Since our purpose is to illustrate the application of the main result obtained in Section [6] the tedious verification of this auxiliary lemma is omitted.

These tool lemmas sort out the relationship between the semicausal trajectory $y_{\xi}(t)$ and the time-delayed trajectory including the initial data. Besides, it can be shown that the assumptions [A1], [A2], and [A3] are satisfied. We assume that the corresponding assumption [A4] is also satisfied. Then we can directly use the causal synthesis formula in Theorem 6.2 and prove the following synthesis result for this differential game problem.

Theorem 7.4 (Closed-Loop Optimal Strategy). For the quadratic differential game of the linear delay system (7.1)-(77.2), the optimal strategy pair $(u, v)$ exists 
uniquely and can be expressed by the following linear state feedback:

$$
\begin{aligned}
\left(\begin{array}{c}
u(t) \\
v(t)
\end{array}\right)=-\{ & \Theta(t, t) y(t)+\sum_{i=1}^{n} \int_{t}^{\min \left(T, t+h_{i}\right)} \Theta(t, \eta) A_{i} y\left(\eta-h_{i}\right) d \eta \\
& +\sum_{i=1}^{n} \int_{\min \left(t, h_{i}\right)}^{h_{i}} \Theta(t, \eta) A_{i} y\left(\eta-h_{i}\right) d \eta \\
& +\sum_{j=1}^{m} \int_{\min \left(t, r_{j}\right)}^{r_{j}} \Theta(t, \eta) B_{i} u\left(\eta-h_{i}\right) d \eta \\
& \left.+\sum_{k=1}^{p} \int_{\min \left(t, \tau_{k}\right)}^{\tau_{k}} \Theta(t, \eta) C_{i} v\left(\eta-h_{i}\right) d \eta\right\}, \quad \text { for } t \in[0, T],
\end{aligned}
$$

where $y(\cdot)$ is the corresponding state function, and

$$
\begin{aligned}
\Theta(t, \eta)= & H(T, t) M F(T-\eta) \\
& +\int_{t}^{T} H(s, t) Q F(s-\eta) d s, \quad(t, \eta) \in[0, T] \times[0, T],
\end{aligned}
$$

with $H(s, t)$ given by (6.4) in which $G$ and $K$ are interpreted by (7.9) and (7.10).

Proof. Directly substitute (7.13) for $y_{t}(s)$ into the causal synthesis formula (6.3), by using (7.5), (7.11), and (7.12) then rearranging terms, we shall obtain (7.14). Again, the detail of verification is omitted.

Corollary 7.5 (Closed-Loop Formula for $t \geq \max \{h, r, \tau\}$ ). The optimal strategy pair for $t \in[\max \{h, r, \tau\}, T]$ is given by the following simpler linear state feedback,

$$
\left(\begin{array}{l}
u(t) \\
v(t)
\end{array}\right)=-\left\{\Theta(t, t) y(t)+\sum_{i=1}^{n} \int_{t}^{\min \left(T, t+h_{i}\right)} \Theta(t, \eta) A_{i} y\left(\eta-h_{i}\right) d \eta\right\} .
$$

\section{Synthesis via Riccati Equation for LINEAR QUADRATIC DIFFERENTIAL GAME}

As the second example, we shall consider the standard linear quadratic differential game described by the linear system

$$
\begin{gathered}
\frac{d y}{d t}=A y(t)+B u(t)+C v(t), \quad t \in[0, T], \\
y(0)=y_{0} \in Y,
\end{gathered}
$$

with the same quadratic performance functional (1.2) in the saddle point sense (1.3). Here the operators $A, B$, and $C$ satisfy the same assumptions made in the above Section 7 But there are no delay terms in this system. Also assume that the assumption [A4] is satisfied. Therefore, as the consequence of Theorem 7.4 in this particular case (with $A_{i}=0, B_{j}=0$, and $C_{k}=0$ for all $i, j$ and $k$ ) we immediately reach the following result. 
Theorem 8.1 (Feedback Optimal Strategy). For this standard linear quadratic differential game problem, the optimal strategy pair exists uniquely and is given by

$$
\left(\begin{array}{l}
u(t) \\
v(t)
\end{array}\right)=-\Theta(t, t) y(t), \quad t \in[0, T],
$$

where the feedback operator is determined by

$$
\begin{gathered}
\Theta(t, t)=\left(\begin{array}{l}
R_{1}^{-1} B^{*} \\
R_{2}^{-1} C^{*}
\end{array}\right)\left\{e^{A(T-t)} M e^{A(T-t)}+\int_{t}^{T} e^{A^{*}(s-t)} Q e^{A(s-t)} d s\right\} \\
+\int_{t}^{T} N_{t}(t, \sigma)\left(\begin{array}{l}
R_{1}^{-1} B^{*} \\
R_{2}^{-1} C^{*}
\end{array}\right)\left\{e^{A^{*}(T-\sigma)} M e^{A(T-\sigma)}+\int_{\sigma}^{T} e^{A^{*}(s-\sigma)} Q e^{A(s-\sigma)} d s\right\} d \sigma,
\end{gathered}
$$

in which $\Theta(t, t)$ comes from (7.15) and $N_{\xi}(t, \sigma)$ is the unique solution of the Fredholm integral equation (5.2) in which

$$
\begin{aligned}
\Pi(t, \sigma)= & {\left[\begin{array}{cc}
R_{1}^{-1} & 0 \\
0 & R_{2}^{-1}
\end{array}\right]\left\{\begin{array}{ll}
B^{*} e^{A^{*}(T-t)} M e^{A(T-\sigma)} B & B^{*} e^{A^{*}(T-t)} M e^{A(T-\sigma)} C \\
C^{*} e^{A^{*}(T-t)} M e^{A(T-\sigma)} B & C^{*} e^{A^{*}(T-t)} M e^{A(T-\sigma)} C
\end{array}\right] } \\
& \left.+\int_{\max (t, \sigma)}^{T}\left[\begin{array}{ll}
B^{*} e^{A^{*}(s-t)} Q e^{A(s-\sigma)} B & B^{*} e^{A^{*}(s-t)} Q e^{A(s-\sigma)} C \\
C^{*} e^{A^{*}(s-t)} Q e^{A(s-\sigma)} B & C^{*} e^{A^{*}(s-t)} Q e^{A(s-\sigma)} C
\end{array}\right] d s\right\} .
\end{aligned}
$$

Proof. This is the translation of Theorem 7.4 in this particular case.

The rest of this section will be devoted to the proof of a reduction of this result to the well-known synthesis of the optimal strategy pair via the differential Riccati equation:

$$
\begin{aligned}
\frac{d}{d t}\langle L(t) y, z\rangle= & -\langle L(t) y, A z\rangle-\langle A y, L(t) z\rangle-\langle Q y, z\rangle \\
& +\left\langle L(t)\left(B R_{1}^{-1} B^{*}+C R_{2}^{-1} C^{*}\right) L(t) y, z\right\rangle, \text { for } y, z \in D(A), t \in[0, T],
\end{aligned}
$$

with the terminal condition

$$
L(T)=M
$$

This synthesis result via the Riccati equation (8.5)-(8.6) can be found in [2] and 9]. What we are going to show is that the Riccati equation (8.5)-8.6) can also be deduced from the approach taken in this paper, namely, from Theorem 8.1 .

For this purpose, we define another operator-valued function $\Xi(t, s): Y \rightarrow Y$ on the region $\{(t, s): 0 \leq s \leq t \leq T\}$ by

$$
\begin{aligned}
& \Xi(t, s)=e^{A(t-s)}-(\Gamma(t), \Delta(t)) W_{s}^{-1}\left(I-P_{s}\right)\left[\begin{array}{cc}
\Gamma^{*} Q & \Gamma_{T}^{*} M \\
\Delta^{*} Q & \Delta_{T}^{*} M
\end{array}\right]\left[\begin{array}{c}
e^{A(\cdot-s)} \\
e^{A(T-s)}
\end{array}\right], \\
& \quad \text { for } 0<s \leq t \leq T ; \\
& \Xi(T, T)=I \text { (identity on } Y) .
\end{aligned}
$$


Lemma 8.2. The operator function $\Xi$ has the following properties.

a) The optimal trajectory $y(\cdot)$ of the quadratic differential game problem for (8.1) satisfies

$$
y(t)=\Xi(t, s) y(s), \text { for } 0<s \leq t \leq T .
$$

b) $\sup \left\{\|\Xi(t, s)\|_{L(Y)}: 0 \leq s \leq t \leq T\right\}<\infty$.

c) $\Xi(t, s)$ is strongly continuous in $t \in[s, T]$ for $0<s \leq T$, and in $s \in(0, t]$ for $0<t \leq T$, respectively.

Proof. The item a) follows from

$$
\begin{gathered}
y(t)=e^{A(t-s)} y(s)+(\Gamma(t), \Delta(t))\left(I-P_{s}\right)\left(\begin{array}{c}
u(t) \\
v(t)
\end{array}\right) \\
{\left[\text { by 4.6] }=e^{A(t-s)} y(s)-(\Gamma(t), \Delta(t)) W_{s}^{-1}\left(I-P_{s}\right)\left[\begin{array}{cc}
\Gamma^{*} Q & \Gamma_{T}^{*} M \\
\Delta^{*} Q & \Delta_{T}^{*} M
\end{array}\right]\left(\begin{array}{c}
y_{\xi} \\
y_{\xi}(T)
\end{array}\right)\right.} \\
=\left\{e^{A(t-s)}-(\Gamma(t), \Delta(t)) W_{s}^{-1}\left(I-P_{s}\right)\left[\begin{array}{cc}
\Gamma^{*} Q & \Gamma_{T}^{*} M \\
\Delta^{*} Q & \Delta_{T}^{*} M
\end{array}\right]\left(\begin{array}{c}
e^{A(\cdot-s)} \\
e^{A(T-s)}
\end{array}\right)\right\} y(s), \text { if } s \neq T .
\end{gathered}
$$

If $s=t=T$, then (8.8) remains valid because $\Xi(T, T)=I$.

The item b) is a consequence of Lemma 3.2 and $\Xi(T, T)=I$.

By (8.7), it is clear that $\Xi(t, s)$ is strongly continuous in $t \in[s, T]$ for $0<s \leq T$. This continuity, the uniform boundedness shown by $b$ ), and the semigroup property of $\Xi(t, s)$ shown by a) all together imply that $\Xi(t, s)$ is strongly continuous in $s \in(0, t]$ for $0<t \leq T$. This implication is a standard argument used in the operator semigroup theory, cf. [7, so the detail is omitted.

Define another operator-valued function $L(\cdot):[0, T] \rightarrow L(Y)$ by

$$
\begin{aligned}
L(t)= & e^{A(T-t)} M e^{A(T-t)}+\int_{t}^{T} e^{A^{*}(s-t)} Q e^{A(s-t)} d s \\
& -\left(D^{*}(t) Q, D_{T}^{*}(t) M\right) E_{t}\left[\begin{array}{cc}
\Gamma & \Delta \\
\Gamma_{T} & \Delta_{T}
\end{array}\right]\left(I-P_{t}\right) \\
& \cdot\left[\begin{array}{cc}
R_{1}^{-1} & 0 \\
0 & R_{2}^{-1}
\end{array}\right]\left[\begin{array}{cc}
\Gamma^{*} \boldsymbol{Q} & \Gamma_{T}^{*} M \\
\Delta^{*} \boldsymbol{Q} & \Delta_{T}^{*} M
\end{array}\right]\left(\begin{array}{c}
e^{A(\cdot-t)} \\
e^{A(T-t)}
\end{array}\right),
\end{aligned}
$$

where the operators

$$
D^{*}(t) \in L\left(L^{2}(0, T ; Y), Y\right)
$$

and

$$
D_{T}^{*}(t) \in L(Y), \quad 0 \leq t \leq T
$$


are defined by

$$
\begin{aligned}
& D^{*}(t) \nu=\int_{t}^{T} e^{A^{*}(s-t)} \nu(s) d s, \text { for } \nu \in L^{2}(0, T ; Y) ; \\
& D_{T}^{*}(t) z=e^{A^{*}(T-t)} z, \text { for } z \in Y .
\end{aligned}
$$

Lemma 8.3. It holds that

$$
\Theta(t, t)=\left(\begin{array}{l}
R_{1}^{-1} B^{*} \\
R_{2}^{-1} C^{*}
\end{array}\right) L(t), t \in[0, T]
$$

Proof. The equality (8.12) follows from (8.3) and (5.1), with the interpretation $G(t, \sigma)=e^{A(t-\sigma)} B$ and $K(t, \sigma)=e^{A(t-\sigma)} C$. The detail steps are shown below.

$$
\begin{aligned}
& \Theta(t, t)=\left(\begin{array}{l}
R_{1}^{-1} B^{*} \\
R_{2}^{-1} C^{*}
\end{array}\right)\left\{e^{A(T-t)} M e^{A(T-t)}+\int_{t}^{T} e^{A^{*}(s-t)} Q e^{A(s-t)} d s\right\} \\
& -\int_{t}^{T}\left(\begin{array}{l}
R_{1}^{-1} B^{*} \\
R_{2}^{-1} C^{*}
\end{array}\right)\left(D^{*}(t) Q, D_{T}^{*}(t) M\right) E_{t}\left(\begin{array}{c}
e^{A(\cdot-\sigma)} \\
e^{A(T-\sigma)}
\end{array}\right)(B, C)\left(I-P_{t}\right) \\
& \cdot\left(\begin{array}{l}
R_{1}^{-1} B^{*} \\
R_{2}^{-1} C^{*}
\end{array}\right)\left\{e^{A(T-\sigma)} M e^{A(T-\sigma)}+\int_{t}^{T} e^{A^{*}(s-\sigma)} Q e^{A(s-\sigma)} d s\right\} d \sigma \\
& =\left(\begin{array}{l}
R_{1}^{-1} B^{*} \\
R_{2}^{-1} C^{*}
\end{array}\right)\left\{e^{A(T-t)} M e^{A(T-t)}+\int_{t}^{T} e^{A^{*}(s-t)} Q e^{A(s-t)} d s\right. \\
& -\left(D^{*}(t) Q, D_{T}^{*}(t)\right) E_{t} \int_{t}^{T}\left(\begin{array}{c}
e^{A(\cdot-\sigma)} \\
e^{A(T-\sigma)}
\end{array}\right)(B, C)\left(I-P_{t}\right) \\
& \left.\cdot\left[\begin{array}{cc}
R_{1}^{-1} & 0 \\
0 & R_{2}^{-1}
\end{array}\right]\left[\begin{array}{cc}
\Gamma^{*} \boldsymbol{Q} & \Gamma_{T}^{*} M \\
\Delta^{*} \boldsymbol{Q} & \Delta_{T}^{*} M
\end{array}\right]\left(\begin{array}{c}
e^{A(\cdot-t)} \\
e^{A(T-t)}
\end{array}\right) d \sigma\right\} \\
& =\left(\begin{array}{l}
R_{1}^{-1} B^{*} \\
R_{2}^{-1} C^{*}
\end{array}\right)\left\{e^{A(T-t)} M e^{A(T-t)}+\int_{t}^{T} e^{A^{*}(s-t)} Q e^{A(s-t)} d s\right. \\
& -\left(D^{*}(t) Q, D_{T}^{*}(t)\right) E_{t}\left[\begin{array}{cc}
\Gamma & \Delta \\
\Gamma_{T} & \Delta_{T}
\end{array}\right]\left(I-P_{t}\right) \\
& \left.\cdot\left[\begin{array}{cc}
R_{1}^{-1} & 0 \\
0 & R_{2}^{-1}
\end{array}\right]\left[\begin{array}{cc}
\Gamma^{*} \boldsymbol{Q} & \Gamma_{T}^{*} M \\
\Delta^{*} \boldsymbol{Q} & \Delta_{T}^{*} M
\end{array}\right]\left(\begin{array}{c}
e^{A(\cdot-t)} \\
e^{A(T-t)}
\end{array}\right) d \sigma\right\} \\
& =\left(\begin{array}{l}
R_{1}^{-1} B^{*} \\
R_{2}^{-1} C^{*}
\end{array}\right) L(t), t \in[0, T] \text {. }
\end{aligned}
$$

Lemma 8.4. $L(\cdot)$ defined by (8.10) can be expressed in terms of $\Xi(\cdot, \cdot)$ by

$$
L(t)=e^{A^{*}(T-t)} M \Xi(T, t)+\int_{t}^{T} e^{A^{*}(s-t)} Q \Xi(s, t) d s, t \in[0, T] .
$$


Proof. This can be checked directly as follows,

$$
\begin{aligned}
& e^{A^{*}(T-t)} M \Xi(T, t)+\int_{t}^{T} e^{A^{*}(s-t)} Q \Xi(s, t) d s \\
& {\left[\text { by (8.7) }=e^{A^{*}(T-t)} M e^{A(T-t)}+\int_{t}^{T} e^{A^{*}(s-t)} Q e^{A(s-t)} d s\right.} \\
& -e^{A^{*}(T-t)} M(\Gamma(T), \Delta(T)) W_{t}^{-1}\left(I-P_{t}\right)\left[\begin{array}{cc}
\Gamma^{*} Q & \Gamma_{T}^{*} M \\
\Delta^{*} Q & \Delta_{T}^{*} M
\end{array}\right]\left(\begin{array}{c}
e^{A(\cdot-t)} \\
e^{A(T-t)}
\end{array}\right) \\
& -\int_{t}^{T} e^{A^{*}(s-t)} M(\Gamma(s), \Delta(s)) W_{t}^{-1}\left(I-P_{t}\right)\left[\begin{array}{cc}
\Gamma^{*} Q & \Gamma_{T}^{*} M \\
\Delta^{*} Q & \Delta_{T}^{*} M
\end{array}\right]\left(\begin{array}{c}
e^{A(\cdot-t)} \\
e^{A(T-t)}
\end{array}\right) d s \\
& {\left[\text { by (8.11) }=e^{A^{*}(T-t)} M e^{A(T-t)}+\int_{t}^{T} e^{A^{*}(s-t)} Q e^{A(s-t)} d s\right.} \\
& -\left(D^{*}(t) Q, D_{T}^{*}(t) M\right)\left[\begin{array}{cc}
\Gamma & \Delta \\
\Gamma_{T} & \Delta_{T}
\end{array}\right] W_{t}^{-1}\left(I-P_{t}\right)\left[\begin{array}{cc}
\Gamma^{*} Q & \Gamma_{T}^{*} M \\
\Delta^{*} Q & \Delta_{T}^{*} M
\end{array}\right]\left(\begin{array}{c}
e^{A(\cdot-t)} \\
e^{A(T-t)}
\end{array}\right) \\
& {\left[\text { by (3.11) }=e^{A^{*}(T-t)} M e^{A(T-t)}+\int_{t}^{T} e^{A^{*}(s-t)} Q e^{A(s-t)} d s\right.} \\
& -\left(D^{*}(t) Q, D_{T}^{*}(t) M\right) E_{t}\left[\begin{array}{cc}
\Gamma & \Delta \\
\Gamma_{T} & \Delta_{T}
\end{array}\right]\left(I-P_{t}\right)\left[\begin{array}{cc}
R_{1}^{-1} & 0 \\
0 & R_{2}^{-1}
\end{array}\right]\left[\begin{array}{cc}
\Gamma^{*} Q & \Gamma_{T}^{*} M \\
\Delta^{*} Q & \Delta_{T}^{*} M
\end{array}\right]\left(\begin{array}{c}
e^{A(\cdot-t)} \\
e^{A(T-t)}
\end{array}\right) \\
& =L(t) \text {. }
\end{aligned}
$$

Thus the proof is completed.

Lemma 8.5. $\Xi(\cdot, \cdot)$ and $L(\cdot)$ also related by

$$
\begin{array}{r}
\Xi(t, s)=e^{A(t-s)}-\int_{s}^{t} \Xi(t, \sigma)\left(B R_{1}^{-1} B^{*}+C R_{2}^{-1} C\right) L(\sigma) e^{A(\sigma-s)} d \sigma, \\
0 \leq s \leq t \leq T .
\end{array}
$$

Proof. Denote by $g_{1}(t)=\Xi(t, s)$ and $g_{2}(t)=$ the right-hand side of the equality (8.14), $t \in[s, T]$. By Lemma 8.2(a), we can verify that $g_{1}(t)$ is a strongly continuous solution of the following Volterra integral equation,

$$
g(t)=e^{A(t-s)}-\int_{s}^{t} e^{A(t-\sigma)}\left(B R_{1}^{-1} B^{*}+C R_{2}^{-1} C\right) L(\sigma) g(\sigma) d \sigma, t \in[s, T] .
$$

Substitute (8.15) for $\Xi(t, s)$ into the expression of $g_{2}(t)$, we can verify that $g_{2}(t)$ is also a strongly continuous solution of the Volterra integral equation (8.15). Then by the uniqueness of the solution of Eq. 8.15 , we conclude that $g_{1}(t) \equiv g_{2}(t)$, so (8.14) holds.

Lemma 8.6. $L(t)$ defined by (8.10) in fact is the unique strongly continuous and self-adjoint solution of the following integral Riccati equation:

$$
\begin{aligned}
L(t)= & e^{A^{*}(T-t)} M e^{A(T-t)} \\
& +\int_{t}^{T} e^{A^{*}(s-t)}\left[Q-L(s)\left(B R_{1}^{-1} B^{*}+C R_{2}^{-1} C\right) L(s)\right] e^{A(s-t)} d s, \quad t \in[0, T] .
\end{aligned}
$$


Proof. First we prove that $L(t)$ satisfies Eq. (8.16). Starting from (8.13),

$$
\begin{gathered}
L(t)=e^{A^{*}(T-t)} M \Xi(T, t)+\int_{t}^{T} e^{A^{*}(s-t)} Q \Xi(s, t) d s \\
{[\text { by }(8.14)]=e^{A^{*}(T-t)} M e^{A(T-t)}+\int_{t}^{T} e^{A^{*}(s-t)} Q e^{A(s-t)} d s} \\
-\int_{t}^{T} e^{A^{*}(T-t)} M \Xi(T, \sigma)\left(B R_{1}^{-1} B^{*}+C R_{2}^{-1} C\right) L(\sigma) e^{A(\sigma-t)} d \sigma \\
-\int_{t}^{T} e^{A^{*}(s-t)} Q \int_{t}^{s} \Xi(s, \sigma)\left(B R_{1}^{-1} B^{*}+C R_{2}^{-1} C^{*}\right) L(\sigma) e^{A(\sigma-t)} d \sigma d s \\
{[\text { by interchanging the integration order in the last term] }} \\
=e^{A^{*}(T-t)} M e^{A(T-t)}+\int_{t}^{T} e^{A^{*}(s-t)} Q e^{A(s-t)} d s \\
-\int_{t}^{T}\left\{e^{A^{*}(T-t)} M \Xi(T, \sigma)+\int_{\sigma}^{T} e^{A^{*}(s-t)} Q \Xi(s, \sigma) d s\right\} \\
\cdot\left(B R_{1}^{-1} B^{*}+C R_{2}^{-1} C^{*}\right) L(\sigma) e^{A(\sigma-t)} d \sigma \\
=e^{A^{*}(T-t)} M e^{A(T-t)}+\int_{t}^{T} e^{A^{*}(s-t)} Q e^{A(s-t)} d s \\
-\int_{t}^{T} e^{A^{*}(\sigma-t)}\left[e^{A^{*}(T-\sigma)} M \Xi(T, \sigma)+\int_{\sigma}^{T} e^{A^{*}(s-\sigma)} Q \Xi(s, \sigma) d s\right] \\
\cdot\left(B R_{1}^{-1} B^{*}+C R_{2}^{-1} C^{*}\right) L(\sigma) e^{A(\sigma-t)} d \sigma \\
\left.-\int_{t}^{T} e^{A^{*}(\sigma-t)} L(\sigma)\left(B R_{1}^{-1} B^{*}+C R_{2}^{-1} C\right) L(\sigma)\right] e^{A(\sigma-t)} d \sigma, \text { for } t \in[0, T] .
\end{gathered}
$$

Therefore, $L(t)$ satisfies Eq. (8.16).

Since (8.10) shows that $L(t)$ is strongly measurable and uniformly bounded, from the right-hand side of (8.16) we see that, for any $y_{0} \in Y, L(t) y_{0}:[0, T] \rightarrow Y$ is strongly continuous. It has been known, cf. [9], that the strongly continuous solution of this integral Riccati equation 8.16) is unique. By transposition, it is seen that $L^{*}(t)$ is also a strongly continuous solution of Eq. (8.16). Hence, $L^{*}(t)=L(t), t \in[0, T]$. The proof is completed.

As a matter of fact, we have proved that the causal synthesis provided by Theorem 8.1 can be reduced to the well-known synthesis result in terms of the solution of the differential Riccati equation (8.5) - This is the following theorem.

Theorem 8.7. For this standard linear-quadratic differential game (8.1)-(1.2), the optimal strategy pair $(u, v)$ exists uniquely and can be expressed by

$$
\left(\begin{array}{l}
u(t) \\
v(t)
\end{array}\right)=-\left(\begin{array}{l}
R_{1}^{-1} B^{*} \\
R_{2}^{-1} C^{*}
\end{array}\right) L(t) y(t), \quad t \in[0, T],
$$

where $L(t)$ is the unique strongly continuous and self-adjoint solution of the differential Riccati equation (8.5)-(8.6), and $y(\cdot)$ is the corresponding trajectory. 
Proof. This result follows directly from Theorem 8.1, Lemma 8.3, Lemma 8.6, and the fact, cf. [9], that a strongly continuous and self-adjoint solution of the integral Riccati equation (8.16) must be the unique strongly continuous and self-adjoint solution of the differential Riccati equation (8.5) $-(8.6)$, and vice versa.

\section{REFERENCES}

1. M. Green and D. J. N. Limebeer, Linear Robust Control, Prentice-Hall, 1995.

2. A. Ichikawa, Linear quadratic differential games in Hilbert spaces, SIAM J. Control and Optimization 14 (1976), 120-136. [MR 52:16681]

3. J.-L. Lions, Some Methods in the Mathematical Analysis of Systems and Their Control, Science Press, Beijing, 1982. MR 84m:49003

4. E. B. Lee and Y. You, Optimal syntheses for infinite dimensional linear delayed stateoutput systems: a semicausality approach, Appl. Math. and Optimization 19 (1989), 113-136. MR 89i:93050

5. E. B. Lee and Y. You, Quadratic optimization for infinite dimensional linear differential difference type systems: synthesis via the Fredholm equations, SIAM J. Control and Optimization 28 (1990), 265-293. MR 91a:49014

6. D. L. Lukes and D. L. Russell, A global theory for linear quadratic differential games, J. Math. Anal. Appl. 33 (1979), 96-123. MR 42:4220

7. A. Pazy, Semigroups of Linear Operators and Applications to Partial Differential equations, Springer-Verlag, New York, 1983. MR 85g:47061

8. A. J. Pritchard and Y. You, Causal feedback optimal control for Volterra integral equations, SIAM J. Control and Optimization 34 (1996), 1874-1890. MR 97k:49035

9. Y. You, Closed-loop syntheses for quadratic differential games of distributed systems, Chinese Ann. Math. 6B (1985), 325-334. MR 87k:90318

10. Y. You, Quadratic differential game of general linear delay systems, J. Math. Pures Appl. 69 (1990), 261-283. MR 92e:90144

Department of Mathematics, University of South Florida, Tampa, Florida 33620

E-mail address: you@math.usf.edu 